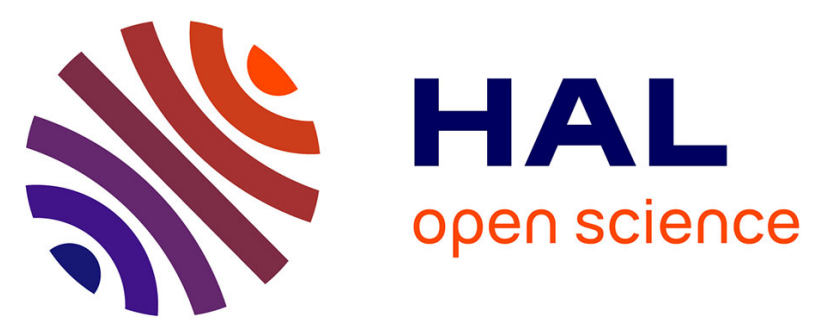

\title{
Characterization of the permittivity of controlled porous water ice-dust mixtures to support the radar exploration of icy bodies
}

\author{
Yann Brouet, Luisa Neves, Pierre Sabouroux, Anny Chantal \\ Levasseur-Regourd, Olivier Poch, Pierre Encrenaz, Antoine Pommerol, \\ Nicolas Thomas, Wlodek Kofman
}

\section{To cite this version:}

Yann Brouet, Luisa Neves, Pierre Sabouroux, Anny Chantal Levasseur-Regourd, Olivier Poch, et al.. Characterization of the permittivity of controlled porous water ice-dust mixtures to support the radar exploration of icy bodies. Journal of Geophysical Research. Planets, 2016, 121 (12), pp.2426-2443. 10.1002/2016JE005045 . insu-01413105

HAL Id: insu-01413105

https://hal-insu.archives-ouvertes.fr/insu-01413105

Submitted on 29 Oct 2020

HAL is a multi-disciplinary open access archive for the deposit and dissemination of scientific research documents, whether they are published or not. The documents may come from teaching and research institutions in France or abroad, or from public or private research centers.
L'archive ouverte pluridisciplinaire HAL, est destinée au dépôt et à la diffusion de documents scientifiques de niveau recherche, publiés ou non, émanant des établissements d'enseignement et de recherche français ou étrangers, des laboratoires publics ou privés. 


\section{Journal of Geophysical Research: Planets}

\section{RESEARCH ARTICLE \\ 10.1002/2016JE005045 \\ Key Points: \\ Characterization of the permittivity of controlled porous water ice-dust mixtures to support the radar exploration of icy bodies}

- We report permittivity measurements between $50 \mathrm{MHz}$ and $2 \mathrm{GHz}$ of porous water ice and ice-dust mixtures with porosities between $31 \%$ and $91 \%$ - The porosity and the dust content dependencies of the real part of the permittivity, which is found to be nondispersive, are determined

- New constraints on the radio waves propagation in porous and icy media, as cometary nuclei, are provided by these measurements

Correspondence to:

Y. Brouet,

yann.brouet@space.unibe.ch

\section{Citation:}

Brouet, Y., L. Neves, P. Sabouroux,

A. C. Levasseur-Regourd, O. Poch,

P. Encrenaz, A. Pommerol, N. Thomas, and W. Kofman (2016), Characterization of the permittivity of controlled porous water ice-dust mixtures to support the radar exploration of icy bodies, $J$. Geophys. Res. Planets, 121, 2426-2443, doi:10.1002/2016JE005045.

Received 30 MAR 2016 Accepted 28 NOV 2016 Accepted article online 8 DEC 2016 Published online 20 DEC 2016

O2016. American Geophysical Union. All Rights Reserved.

\author{
Y. Brouet ${ }^{1}$ iD, L. Neves ${ }^{2}$ iD , P. Sabouroux ${ }^{2}$, A. C. Levasseur-Regourd ${ }^{3}$ D, O. Poch ${ }^{1}$ (D) P. Encrenaz ${ }^{4}$, \\ A. Pommerol ${ }^{1}$, N. Thomas ${ }^{1}$ iD, and W. Kofman ${ }^{5,6}$ (D) \\ ${ }^{1}$ Physics Institute, University of Bern, Bern, Switzerland, ${ }^{2}$ Aix-Marseille Université, CNRS, Centrale Marseille, Institut Fresnel, \\ UMR 7249, Campus Universitaire de Saint-Jérôme, Marseille, France, ${ }^{3}$ UPMC (Sorbonne University); UVSQ (UPSay); \\ CNRS/INSU; LATMOS-IPSL, Paris, France, ${ }^{4}$ LERMA, Observatoire de Paris, Paris, France, 5 UJF-Grenoble 1/CNRS-INSU, IPAG, \\ UMR, Grenoble, France, ${ }^{6}$ Space Research Centre, PAS, Warsow, Poland
}

Abstract The internal properties of porous and icy bodies in the solar system can be investigated by ground-penetrating radars (GPRs), like the COmet Nucleus Sounding Experiment by Radiowave Transmission instrument on board the Rosetta spacecraft which has sounded the interior of the nucleus of comet 67P/Churyumov-Gerasimenko. Accurate constraints on the permittivity of icy media are needed for the interpretation of the data. We report novel permittivity measurements performed on water ice samples and icy mixtures with porosities in the 31-91\% range. The measurements have been performed between $50 \mathrm{MHz}$ and $2 \mathrm{GHz}$ with a coaxial cell on a total of 38 samples with a good reproducibility. We used controlled procedures to produce fine-grained and coarse-grained ice samples with a mean diameter of $4.5 \mu \mathrm{m}$ and $67 \mu \mathrm{m}$, respectively, and to prepare icy mixtures. The JSC-1A lunar regolith simulant was used as the dust component in the mixtures. The results are focused on the real-part $\epsilon^{\prime}$ of the permittivity, which constrains the phase velocity of the radio waves in low-loss media. The values of $\epsilon^{\prime}$ show a nondispersive behavior and are within the range of 1.1 to 2.7. They decrease with the increasing porosity $\Phi$ according to $E^{(1-\Phi)}$, with $E$ equal to about 3.13 for pure water ice, and in the 3.8-7.5 range for ice-dust mixtures with a dust-to-ice volumetric ratio in the $0.1-2.8$ range, respectively. These measurements are also relevant for radiometers operating in the millimeter-submillimeter domains, as suggested by the nondispersive behavior of the mixtures and of the pure components.

\section{Introduction}

Comets are porous and icy media composed of mostly water and carbon dioxide ices and dust. The past space missions dedicated to the "in situ" investigations of cometary nuclei for three decades have provided constraints on their low bulk density estimated between 100 and $1200 \mathrm{~kg} \mathrm{~m}^{-3}$ [Festou et al., 2004; Barucci et al., 2011, and references therein]. The Rosetta spacecraft, of the European Space Agency, successfully began its rendezvous with comet 67P/Churyumov-Gerasimenko (hereafter 67P) in August 2014. Sierks et al. [2015] have provided a first estimation of the bulk density and the porosity (i.e., the volume fraction of empty space in a given volume) of the whole nucleus in the range of 420 to $520 \mathrm{~kg} \mathrm{~m}^{-3}$ and of 70 to $80 \%$, respectively. These values have been refined by modeling of the gravity field by Pätzold et al. [2016]. Comets are not the only porous and icy objects in the solar system. Investigations on transneptunian objects and some asteroids of the main belt have also revealed such properties [Barucci et al., 2008; Cartwright, 2007]. These small bodies may be representative of the most primitive objects, scarcely processed, of the solar system from which planets were formed. Therefore, investigations of their internal properties may provide constraints on the protosolar nebula and on planetary formation models.

The ground-penetrating radar (hereafter GPR) technique is very well adapted to the investigation of the internal properties of icy bodies in the solar system because of the transparency of ice to radio waves at frequencies in the $\mathrm{MHz}$ range [Waite and Schmidt, 1962]. As radio waves interact with the different materials encountered at depth, GPRs can provide an electromagnetic image of subsurface structures. The GPR performance, related to the electromagnetic behavior of materials present in the propagation path, is controlled by the relative complex permittivity $\epsilon$ (hereafter permittivity) [e.g., Kofman et al., 2010; Ulaby and Long, 2014]. The permittivity 
is separated into two components: $\epsilon^{\prime}+i \epsilon^{\prime \prime}$. The real part of the permittivity, $\epsilon^{\prime}$, is inversely proportional to the square of the phase velocity of the radio wave for low-loss media and describes how the material stores and reradiates energy. The imaginary part of the permittivity, $\epsilon^{\prime \prime}$, describes the energy dissipation process associated with wave propagation and diffusion inside the material. The permittivity varies with the frequency of measurements as well as the temperature, the composition, and the porosity of the medium [e.g., Campbell and Ulrichs, 1969; Ulaby and Long, 2014]. Thus, the characterization of the permittivity as a function of these parameters is necessary to interpret properly data collected by GPRs dedicated to planetary exploration. The CONSERT (COmet Nucleus Sounding Experiment by Radiowave Transmission) instrument on board the Rosetta spacecraft was the first GPR dedicated to the sounding of a cometary nucleus [Kofman et al., 1998]. During the First Science Sequence (hereafter FSS) of measurement, between 12 and 13 November 2014, it has used radio waves at a center-band frequency equal to $90 \mathrm{MHz}$ to sound the small lobe of the cometary nucleus 67P [Kofman et al., 2015; Ciarletti et al., 2015].

The permittivity of pure solid water ice as a function of frequency and temperature is well documented [e.g., Johari and Whalley, 1981; Mätzler and Wegmüller, 1987; Pettinelli et al., 2015]. The permittivity spectrum of pure water ice is well described by the Debye relaxation model from $\mathrm{Hz}$ to several hundreds of $\mathrm{MHz}$ [Johari and Whalley, 1981] even though at a temperature below $250 \mathrm{~K}$, it is better described by a Cole-Cole model [Cole and Cole, 1941; von Hippel et al., 1971]. The relaxation frequency of solid water ice occurs in the hertz-kilohertz region as a function of the temperature [see Pettinelli et al., 2015, Figure 10]. In the frequency range of $1 \mathrm{MHz}$ to $10 \mathrm{GHz}$, Gough [1972] and Mätzler and Wegmüller [1987] showed that the real part of the permittivity of solid water ice is essentially frequency independent and has a value in the $3.1-3.2$ range with a slight temperature dependence. The imaginary part of the permittivity of solid water ice is lower than 0.001 [Mätzler et al., 2006]. Regarding water ice-dust mixtures, laboratory permittivity measurements are very limited and relatively recent [Hérique et al., 2002; Stillman et al., 2010; Heggy et al., 2012; Mattei et al., 2014]. Indeed, no measurements on ice-dust mixtures with a porosity greater than $50 \%$ are reported in the literature over the whole frequency range of the radio domain. Pettinelli et al. [2015] also point out that the lack of a standard procedure in sample preparation and measuring methodology raises some questions about the reliability of experimental data obtained. Moreover the porosity of samples is a key parameter to take into account during the measurements of the dielectric properties [Brouet et al., 2014, 2015], but it is not always well documented. Experimental data regarding samples with a porosity larger than $50 \%$ are needed for radar data inversion of very porous media, such as cometary nuclei and particularly the nucleus of comet 67P sounded by the CONSERT instrument.

The scope of the current paper is to describe standard procedures to prepare and measure the permittivity of pure water ice samples and ice-dust mixtures with a high range of porosity from about $31 \%$ to $91 \%$ over a frequency range relevant for GPRs. We present two procedures to prepare pure water ice samples composed of quasi-spherical particles and a procedure to prepare different ice-dust mixtures with variations of the dust-to-ice volumetric ratio and of the porosity. We selected two samples of JSC-1A lunar regolith simulant as the dust components for the mixtures. The JSC-1A simulant is composed of silicate-rich grains with an original grain size distribution below $1 \mathrm{~mm}$. It is relevant to reproduce the properties of the lunar regolith. In addition, its mafic composition and its size distribution make it suitable for radar investigations dedicated to Mars [e.g., Mattei et al., 2014] and asteroids [e.g., Palmer et al., 2015]. It has also a porous structure [Brouet et al., 2015], which can be interesting to approach, for example, the fluffy structure of the cometary dust, estimated from in situ measurements [e.g., Fulle et al., 2000, 2016] and from remote light scattering observations [e.g., Levasseur-Regourd et al., 2009]. The method used for the permittivity measurements over the frequency range of $50 \mathrm{MHz}$ to $2 \mathrm{GHz}$ is then defined. New results on the real part of the permittivity of porous water ice and ice-dust mixtures are presented, with an emphasis on the porosity and the dust-to-ice volumetric ratio dependencies. The reproducibility of these measurements is also discussed. Finally, the results are compared to previous results available in the literature obtained on water ice and ice-dust mixtures, and an empirical formula describing the porosity and the dust content dependencies of the real part of the permittivity, derived from these experimental data, is proposed.

\section{Samples Preparation}

\subsection{Pure Water Ice}

We prepared samples of pure and porous water ice by using the SPIPA (Setup for the Production of Icy Planetary Analogs) facility developed in the LOSSy laboratory at the University of Bern. Two different techniques 


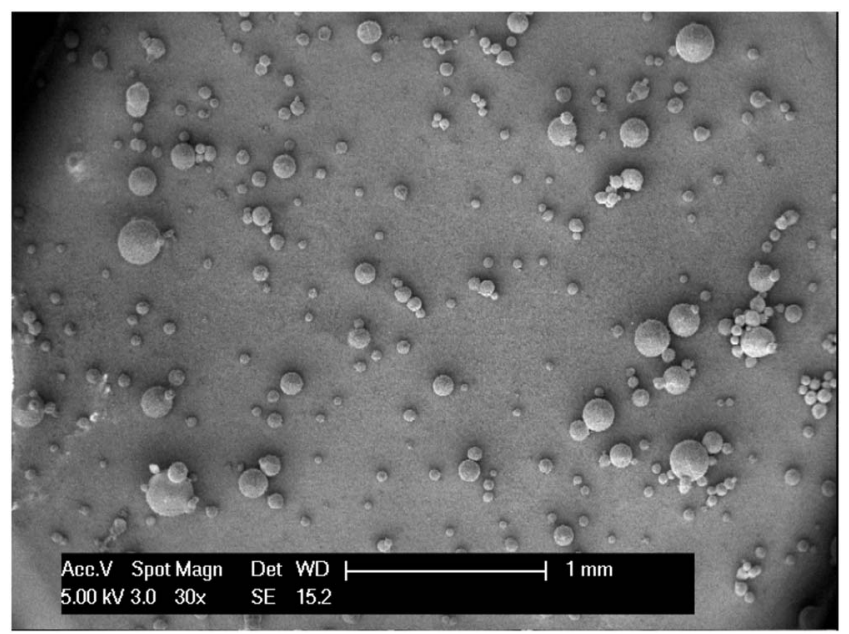

Figure 1. Scanning electron microscope image of fresh water ice particles with a mean diameter equal to $67 \pm 31 \mu \mathrm{m}$. were used to produce two different particle size distributions of quasi-spherical ice particles with a type Ih crystalline structure [Bartels-Rausch et al., 2012]. The solid density $\rho_{\text {I }}$ of the Ih crystalline water ice particles is equal to $917 \mathrm{~kg} \mathrm{~m}^{-3}$ [e.g., Lide, 2005].

A first technique uses an ultrasonic nebulizer in order to form very fine droplets of deionized water. These droplets are conducted through a plastic tube inside a modified chest freezer cooled down to about $223 \mathrm{~K}$ and are then deposited onto an aluminum bowl in contact with a vessel containing liquid nitrogen [Yoldi et al., 2015; Jost et al., 2016]. This technique produces quasi-spherical ice particles with a mean diameter equal to $4.5 \pm 2.5 \mu \mathrm{m}$ (the second number is the

standard deviation) as measured by cryo-SEM (Scanning Electron Microscope) [see Jost et al., 2016, Figure 5.a]. A second technique consists of using a peristaltic pump to conduct deionized water through a $2 \mathrm{~mm}$ diameter tube connected to a Hielscher $200 \mathrm{Ht}$ ultrasonic unit equipped with a S26d18S sonotrode. The pumped water is injected into the sonotrode where it spreads out as a thin film on the nozzle surface. The ultrasonic vibrations delivered to the sonotrode nozzle disintegrate the liquid film into microdroplets. This nebulization takes place inside the same chest freezer as in the first technique. The droplets ejected by the sonotrode nozzle fall into an aluminum bowl containing liquid nitrogen and in contact with the vessel containing liquid nitrogen. Then the droplets rapidly freeze into quasi-spherical ice particles, as showed in Figure 1. The size distribution analysis by cryo-SEM of the ice particles suggests a mean diameter of $67 \pm 31 \mu \mathrm{m}$ (the second number is the standard deviation) [Yoldi et al., 2015; Poch et al., 2016].

Ice particles produced by the first technique will be referred to as fine-grained ice, with a mean diameter of $4.5 \pm 2.5 \mu \mathrm{m}$, and those produced by the second technique will be referred to as coarse-grained ice, with a mean diameter of $67 \pm 31 \mu \mathrm{m}$, in the rest of this paper. A sample of pure solid ice was also prepared with deionized water used to produce the ice particles described above.

\subsection{Mixtures: Pure Water Ice and JSC-1A Lunar Regolith Simulant}

The JSC-1A lunar regolith simulant is composed of volcanic ashes with a basaltic composition [Hill et al., 2007] collected in the Merriam Crater's south flank in Arizona (USA). Alshibli and Hasan [2009] estimated the mean solid density of the JSC-1A simulant to be equal to $2920 \mathrm{~kg} \mathrm{~m}^{-3}$. We used two samples of JSC-1A lunar regolith simulant (hereafter also defined as dust) to prepare homogeneous and porous mixtures with different dust-to-ice volumetric ratios. The permittivity of several subsamples of the JSC-1A simulant with different size ranges covering a few $\mu \mathrm{m}$ to $500 \mu \mathrm{m}$ have been characterized by Brouet et al. [2015] in the same range of measurement frequencies. The measurements of the different noncompacted JSC-1 A samples show a nondispersive behavior and a slight linear decrease of $\epsilon^{\prime}$ with the increasing porosity, allowing to estimate $\epsilon^{\prime}$ for an ice-free dust medium with high porosities at the operating frequency of the CONSERT radar on board the Rosetta spacecraft.

The fine-grained ice samples were mixed with a JSC-1A sample with a mean grain size equal to $24 \pm 9 \mu \mathrm{m}$, referred to as JSC-1 A\#1 in Brouet et al. [2015]. The coarse-grained ice samples were mixed with a JSC-1A sample with a mean grain size equal to $78 \pm 28 \mu \mathrm{m}$, referred to as JSC-1A\#2 in the same reference. The first and second mixtures mentioned above will be referred to as the fine-grained mixture and coarse-grained mixture, respectively, in the rest of this paper. We prepared fine-grained mixtures with dust-to-ice volumetric ratios equal to $0.10,0.31,1.26$, and 2.83 and coarse-grained mixtures with a dust-to-ice volumetric ratio equal to 0.31. All mixtures were prepared with the same well-defined procedure. First, we collect the mass of dust and the mass of fresh ice needed to obtain the expected dust-to-ice volumetric ratio by using a precision weighing balance $( \pm 0.001 \mathrm{~g})$. The fresh ice sample is then stored in an aluminum bottle at the temperature of the chest 


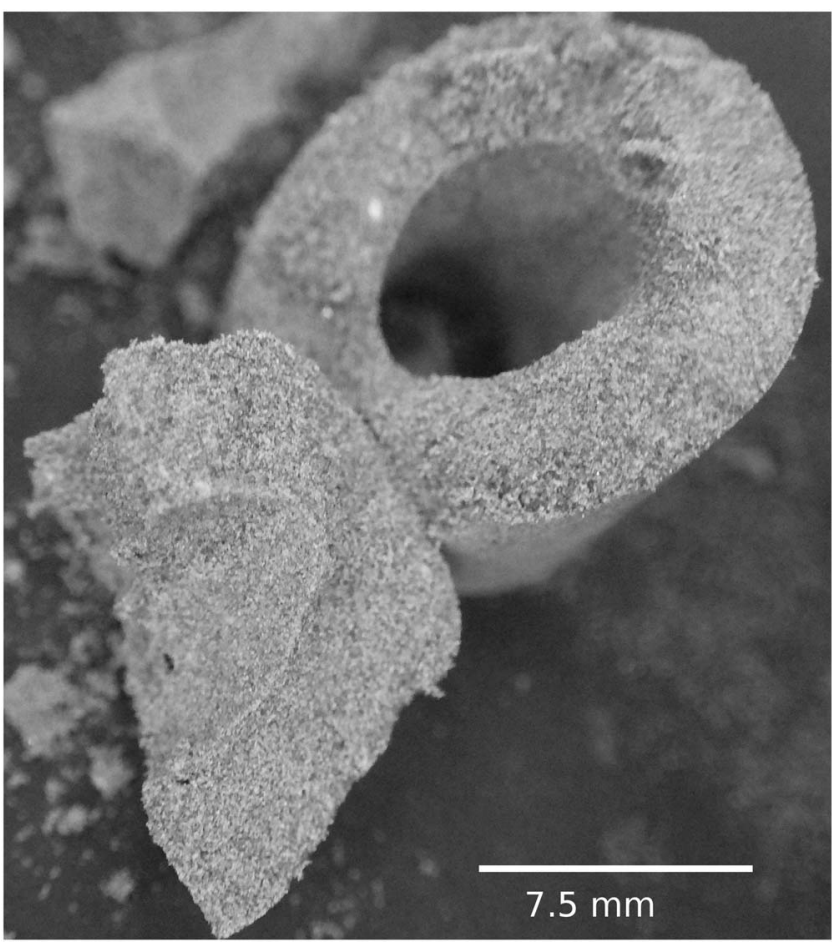

Figure 2. Homogeneous mixture composed of a JSC-1A simulant sample with a mean grain size equal to $24 \pm 9 \mu \mathrm{m}$ and fine-grained ice. This picture was taken after having extracted the sample from the sample holder after the measurements. freezer (about $220 \mathrm{~K}$ ). Second, possible adsorbed moisture is removed from the dust sample, in order to avoid interactions with radio waves and an increase of the real and imaginary parts of the permittivity [Sabouroux and $B a, 2011$ ]. The samples are dried by using a moisture analyzer which provides an estimation of their volumetric moisture content (found to be equal or less than $0.2 \%$ ). After that, the dust sample is stored about $15 \mathrm{~min}$ in the same chest freezer as the fresh ice, which provides a relatively dry environment and cools down the dust sample. The ice and dust samples are then mixed within an aluminum bottle over a vortex shaker in several mixing steps. First, the pure components of the mixing are mixed by keeping the ratio of masses around 1 , and then the successive products of the mixing process are also mixed with a pure component by keeping the ratio of masses around 1 . Any residue is mixed at the end of the process to obtain the final mixture. For example, in order to prepare a sample with a dust-to-ice volumetric ratio equal to 0.1 , i.e., a dust mass fraction equal to 0.25 , we prepared $2.520 \mathrm{~g}$ of JSC- $1 \mathrm{A \#} 1$ simulant and $7.584 \mathrm{~g}$ of fine-grained ice. A first step of mixing is performed with a ratio of masses around $1: 2.520 \mathrm{~g}$ of JSC-1A\#1 simulant mixed with $2.754 \mathrm{~g}$ of ice; and then a second step of mixing, again with a ratio of masses around 1, provides a mixture with the desired final dust mass fraction: $5.274 \mathrm{~g}$ of material obtained from the first step is mixed with the rest of the water ice, i.e., $4.830 \mathrm{~g}$. The duration of the mixing process is always the same and the aluminum bottle is repeatedly cooled down in liquid nitrogen. This reproducible technique provides homogeneous samples, as showed in Figure 2 and discussed in section 4.1, of ice-dust mixtures with controlled dust-to-ice volumetric ratio.

\section{Instrumentation}

\subsection{Measurements With a Conical Coaxial Cell Between $50 \mathrm{MHz}$ and $2 \mathrm{GHz}$}

Measurements of the real part of the permittivity between $50 \mathrm{MHz}$ and $2 \mathrm{GHz}$ on the different pure ices and icy mixtures were performed in 2015 at the University of Bern (Switzerland). All the measurements were performed at about $243 \mathrm{~K}$ and under ambient pressure. We used the same experimental setup with which measurements of the bulk density and the porosity dependencies of the real part of the permittivity were performed between $50 \mathrm{MHz}$ and $6 \mathrm{GHz}$ by Brouet et al. [2015] for volcanic ashes collected in Mont Etna (Italy) and JSC-1A lunar regolith simulant.

As showed in Figure 3 the experimental setup consists of a coaxial cell connected to a vector network analyzer (VNA, Anritsu Master MS2038C). The coaxial cell is equipped with a sample holder which consists of a central containment area of sample, transition lines, and connectors, for which the dimensions are well known. Two dielectric walls in PolyTetraFluoroEthylene (PTFE) delimit the containment area. The phase and amplitude of the complex scattering parameters $S_{11}$ and $S_{22}$ (i.e., the reflection coefficients measured at ports 1 and 2 of the coaxial cell, respectively), and the complex scattering parameters $S_{21}$ and $S_{12}$ (i.e., the transmission coefficients measured at ports 2 and 1 of the coaxial cell, respectively) of the cell loaded with the sample under test are measured with a calibrated VNA over 50 frequencies between $50 \mathrm{MHz}$ and $2 \mathrm{GHz}$ [Nicolson and Ross, 1970; Georget et al., 2014]. The calibration of the VNA was performed before each measurement on a given 

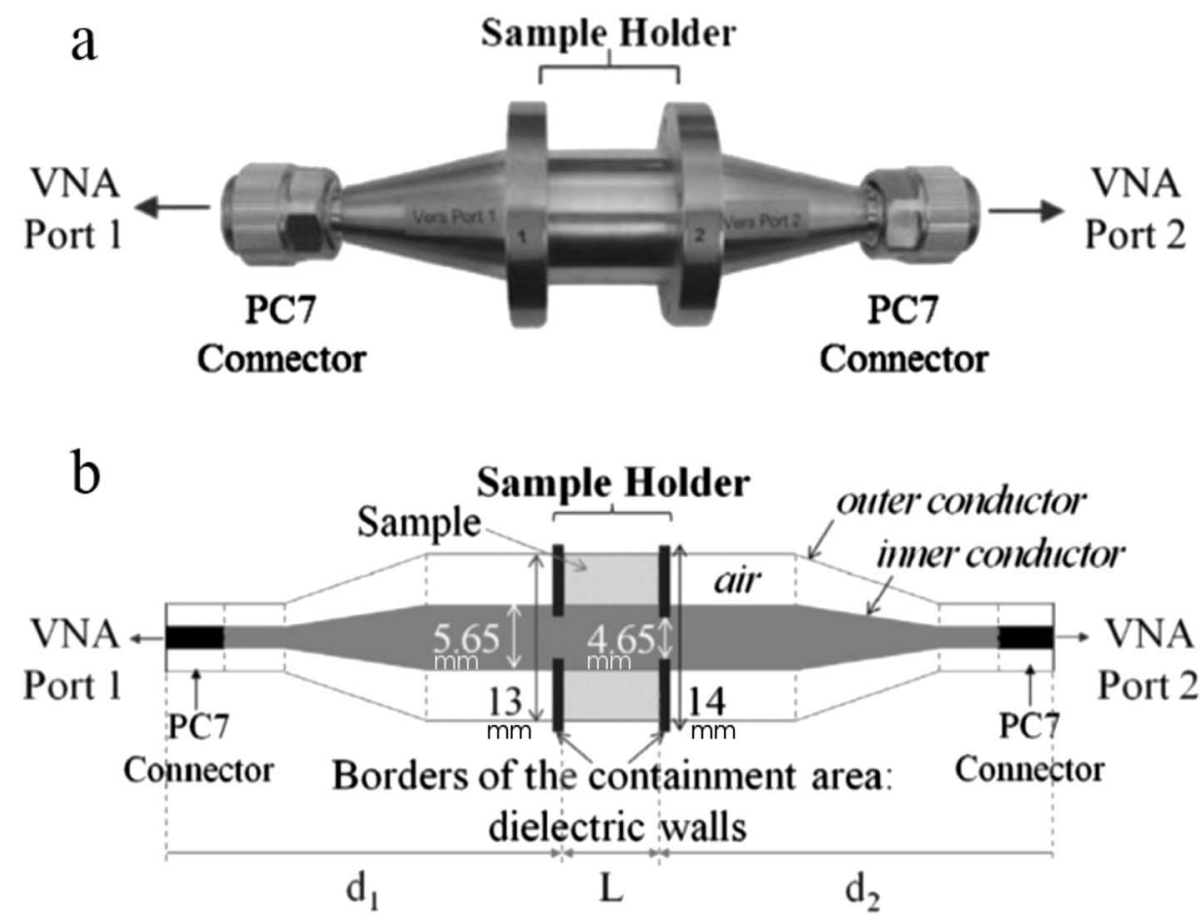

Figure 3. (a) Image and (b) schematic of the conical coaxial cell used to perform the permittivity measurements between $50 \mathrm{MHz}$ and $2 \mathrm{GHz}$ [from Brouet et al., 2015].

sample with a full SOLT (Short-Open-Load-Through). A first step of "deembedding" allows the determination of the sample scattering parameters, taking into account the phase propagation term and the attenuation propagation term of the coaxial cell, as well as the slight mismatching of impedance added by the presence of the two dielectric walls [Georget et al., 2014]. Then the permittivity at a given frequency is determined by applying the Nicolson-Ross procedure [Nicolson and Ross, 1970] to the sample scattering parameters $S_{11}$ and $S_{21}$, on the one hand, and to $S_{22}$ and $S_{12}$, on the other hand. Then two permittivity spectra for each sample are presented and discussed in section 4.1. More details on this setup and procedure of measurements are presented in Georget et al. [2014].

Compared to a straight coaxial line, the original design of the cell improves the handling, containment, and positioning of the sample and allows to accurately define a protocol of sample holder and cell preparation. This protocol, presented in section 3.2, has been precisely followed for all samples. Thanks to this protocol and the reproducible methods of sample preparation previously mentioned, the measurements are reproducible, as discussed in section 4.4. Although this setup is particularly well adapted for reproducible and accurate measurements of the real part of the permittivity of porous pure water ices and icy mixtures, the measurement of the imaginary part of the permittivity of porous icy samples is very challenging because of the very low magnitude of dielectric losses and the sensitivity limit of the instrument which is reached with an imaginary part lower than 0.1. Therefore, the results are focused on the real part of the permittivity.

\subsection{Sample Holder and Cell Preparation at Low Temperature}

The introduction of the porous icy samples inside the sample holder, as presented in Figure 4a, is performed in a chest freezer. The sample holder is installed vertically on an aluminum plate in direct contact with a copper lid covering a stainless steel vessel filled with liquid nitrogen. The temperature of the aluminum plate is stable around $200 \mathrm{~K}$. All tools dedicated to the handling of the icy samples (i.e., spoons, metallic sticks, and fine brushes) and the sample holders are first cooled down in the freezer. The icy samples are introduced inside the sample holder by using a fine brush or with a metallic cylinder machined to fit to the dimensions of the sample holder. Different pressures are applied in order to obtain different compaction, resulting in a variation of the bulk density and the porosity. For all measurements, the mass of the sample holder filled with the sample is determined in order to estimate the bulk density of the sample, knowing that the filled volume is equal to $1.291 \pm 0.288 \mathrm{~cm}^{3}$. The sample holder is first covered by an isolating foam inside the freezer before the 

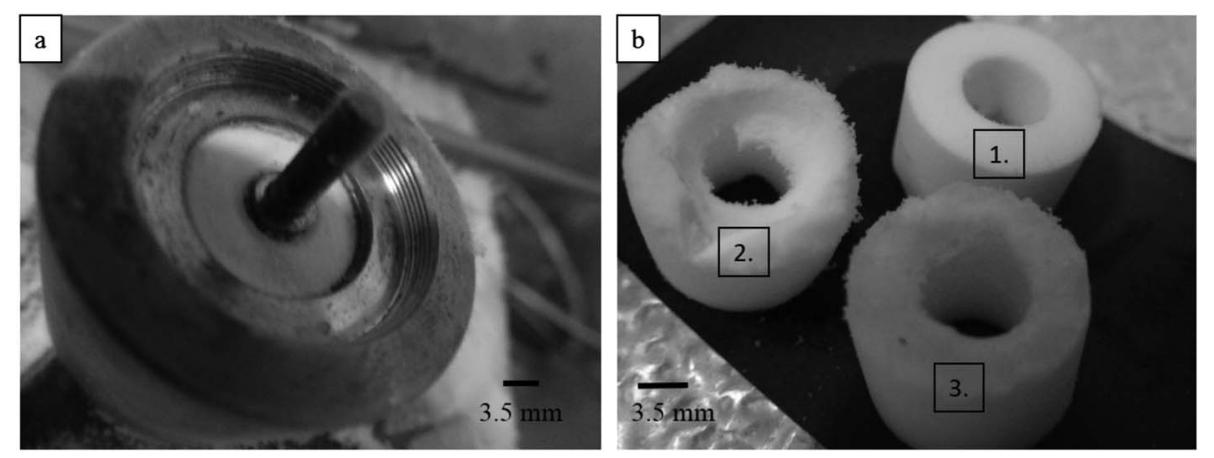

Figure 4. (a) Sample holder filled by pure water ice particles. (b) Samples of pure water ice extracted from the sample holder after the measurements, at a temperature around $243 \mathrm{~K}$, with different macroscopic aspects due to their different values of porosity: 1 . low porosity ( $\backsim 50 \%) ; 2$. high porosity $(\backsim 85 \%) ; 3$. intermediate porosity $(\backsim 65 \%)$.

weighing, which takes place outside the freezer, in order to avoid any condensation of water. The uncertainties on the bulk density, obtained with the method of error propagation, are in the range of 8 to $27 \mathrm{~kg} \mathrm{~m}^{-3}$, as showed in Tables 1 and 2 . For pure water ice samples, the porosity $\Phi$ can then be determined by $\left(1-\rho / \rho_{l}\right)$, where $\rho$ is the bulk density of the sample and $\rho_{l}$ is the solid density of the pure water ice. The porosity of a noncompacted sample of fine-grained ice is likely to be near or more than $70 \%$, whereas it is likely near or less than $50 \%$ for a noncompacted sample of coarse-grained ice. The properties of the samples are summarized in Table 1. The porosity $\Phi$ of the mixture samples can be determined, knowing the dust mass fraction $F_{D}$, the solid density of the dust $\rho_{D}$, and the ice $\rho_{l}$, by the following equation:

$$
\Phi=1-\rho \times\left(\frac{F_{D}}{\rho_{D}}+\frac{1-F_{D}}{\rho_{l}}\right) ;
$$

which is derived from the relation between the porosity and the bulk density mentioned above. The properties of the samples are summarized in Table 2. The uncertainties on the porosity, also obtained with the method of error propagation, are in the range of $0.6 \%$ to $1.7 \%$.

The loading of the sample holder in the cell occurs directly after the first weighing and takes place in another freezer at a stable temperature around $243 \mathrm{~K}$. Before the loading, the interior of the cell is carefully cleaned with

Table 1. Main Characteristics of the Porous and Pure Water Ice Samples Prepared for the Permittivity Measurements With the Two Methods Described in Section $2.1^{\mathrm{a}}$

\begin{tabular}{lccccc} 
Sample & $\#$ & $\phi \pm \sigma_{\phi}(\mu \mathrm{m})$ & $m \pm 10(\mathrm{mg})$ & $\rho \pm \delta \rho\left(\mathrm{kg} \mathrm{m}^{-3}\right)$ & $\Phi \pm \delta \Phi(\%)$ \\
\hline Fine-grained ice & 1 & & 230 & $178 \pm 9$ & $80.6 \pm 0.9$ \\
& 2 & & 480 & $372 \pm 11$ & $59.5 \pm 1.2$ \\
& 3 & & 599 & $464 \pm 13$ & $49.4 \pm 1.4$ \\
& 4 & $4.5 \pm 2.5$ & 170 & $132 \pm 8$ & $85.6 \pm 0.9$ \\
& 5 & & 280 & $217 \pm 9$ & $76.3 \pm 1.0$ \\
& 6 & 410 & $318 \pm 10$ & $65.4 \pm 1.1$ \\
& 7 & 510 & $395 \pm 12$ & $56.9 \pm 1.3$ \\
& 8 & 600 & $465 \pm 13$ & $49.3 \pm 1.4$ \\
& 9 & & 600 & $465 \pm 13$ & $49.3 \pm 1.4$ \\
& 10 & & 790 & $612 \pm 16$ & $33.3 \pm 1.7$ \\
& 11 & $67 \pm 31$ & 800 & $620 \pm 16$ & $32.4 \pm 1.7$ \\
& 12 & & 810 & $627 \pm 16$ & $31.6 \pm 1.7$ \\
\hline
\end{tabular}

${ }^{a}$ With $\phi$, the mean grain size and $\sigma_{\phi}$ its standard deviation; $m$, the mass of sample inside the sample holder; $\rho$, the bulk density and $\delta \rho$ its uncertainty; $\Phi$, the porosity and $\delta \Phi$ its uncertainty. The uncertainties on the bulk density and the porosity values have been assessed by using the method of error propagation. The samples \#1-3 and \#4-8 were prepared and measured in October and in December 2015, respectively. The samples \#9-12 were prepared and measured in December 2015. 
Table 2. Main Characteristics of the Icy Mixtures Prepared for the Permittivity Measurements ${ }^{\mathrm{a}}$

\begin{tabular}{|c|c|c|c|c|c|c|c|}
\hline Sample & $F_{D}$ & $V_{D / I}$ & $\#$ & $\phi \pm \sigma_{\phi}(\mu \mathrm{m})$ & $m \pm 10(\mathrm{mg})$ & $\rho \pm \delta \rho\left(\mathrm{kg} \mathrm{m}^{-3}\right)$ & $\Phi \pm \delta \Phi(\%)$ \\
\hline \multirow[t]{21}{*}{ Fine-grained mixture } & & & 14 & & 135 & $105 \pm 8$ & $90.6 \pm 0.7$ \\
\hline & & & 15 & & 258 & $200 \pm 9$ & $81.9 \pm 0.8$ \\
\hline & & & 16 & & 260 & $201 \pm 9$ & $81.8 \pm 0.8$ \\
\hline & 0.25 & 0.10 & 17 & & 266 & $206 \pm 9$ & $81.4 \pm 0.8$ \\
\hline & & & 18 & & 600 & $464 \pm 13$ & $58.1 \pm 1.2$ \\
\hline & & & 19 & & 202 & $156 \pm 8$ & $88.7 \pm 0.6$ \\
\hline & & & 20 & & 269 & $208 \pm 9$ & $84.9 \pm 0.7$ \\
\hline & 0.49 & 0.31 & 21 & & 320 & $248 \pm 10$ & $82.1 \pm 0.7$ \\
\hline & & & 22 & & 450 & $349 \pm 11$ & $74.8 \pm 0.8$ \\
\hline & & & 23 & & 640 & $496 \pm 14$ & $64.1 \pm 1.0$ \\
\hline & & & & $4.5 \pm 2.5$ (ice), $24 \pm 9$ (JSC-1A\#1) & & & \\
\hline & & & 24 & & 601 & $466 \pm 13$ & $77.1 \pm 0.6$ \\
\hline & & & 25 & & 672 & $521 \pm 14$ & $74.4 \pm 0.7$ \\
\hline & 0.80 & 1.26 & 26 & & 754 & $584 \pm 15$ & $71.3 \pm 0.7$ \\
\hline & & & 27 & & 830 & $643 \pm 16$ & $68.4 \pm 0.8$ \\
\hline & & & 28 & & 1018 & $789 \pm 19$ & $61.2 \pm 1.0$ \\
\hline & & & 29 & & 989 & $766 \pm 19$ & $68.0 \pm 0.8$ \\
\hline & 0.90 & 2.83 & 30 & & 1057 & $819 \pm 20$ & $65.8 \pm 0.8$ \\
\hline & & & 31 & & 1169 & $905 \pm 22$ & $62.2 \pm 0.9$ \\
\hline & & & 32 & & 1280 & $991 \pm 23$ & $58.6 \pm 1.0$ \\
\hline & & & 33 & & 1515 & $1174 \pm 27$ & $51.0 \pm 1.2$ \\
\hline \multirow[t]{6}{*}{ Coarse-grained mixture } & & & 34 & & 896 & $694 \pm 17$ & $49.8 \pm 1.3$ \\
\hline & & & 35 & & 920 & $713 \pm 18$ & $48.4 \pm 1.3$ \\
\hline & 0.49 & 0.31 & 36 & $67 \pm 31$ (ice), $74 \pm 28$ (JSC-1A\#2) & 963 & $746 \pm 18$ & $46.0 \pm 1.3$ \\
\hline & & & 37 & & 975 & $755 \pm 19$ & $45.3 \pm 1.3$ \\
\hline & & & 38 & & 1023 & $792 \pm 19$ & $42.6 \pm 1.4$ \\
\hline & & & 39 & & 1033 & $800 \pm 19$ & $42.1 \pm 1.4$ \\
\hline
\end{tabular}

${ }^{\mathrm{a}} F_{D}$, the dust mass fraction of mixtures; $V_{D / l}$, the dust-to-ice volumetric ratio of mixtures; $\phi$, the mean grain size, and $\sigma_{\phi}$ its standard deviation; $m$, the mass of sample inside the sample holder; $\rho$, the bulk density and $\delta \rho$ its uncertainty; $\Phi$, the porosity and $\delta \Phi$ its uncertainty. The uncertainties on the bulk density and the porosity values have been assessed by using the method of error propagation. The samples were prepared and measured in December 2015.

an absorbent paper to remove possible water which may have condensed and could affect the measurements. The temperature of the cell is monitored by a PT100 temperature sensor attached to the cell. Then, after a dozen minutes needed to stabilize the temperature of the cell, it is first covered by an isolating foam and, second, brought to a measurement table where it is connected to the VNA. After the measurements, the cell is disassembled inside the freezer and the sample holder is weighted again in order to check that the state of the sample is stable. Figure $4 \mathrm{~b}$ shows the macroscopic aspect of samples with different porosities extracted from the sample holders after the permittivity measurements.

\subsection{Measurement Errors}

The values of $\epsilon^{\prime}$ obtained from the two pairs of scattering parameters in the frequency band from $50 \mathrm{MHz}$ to $2 \mathrm{GHz}$ and taken into account in section 4, correspond to the mean real part of the permittivity derived from 10 acquisitions of the scattering parameters performed successively. The absolute errors on each measurement of a given subsample are estimated to be the sum of standard deviation and experimental error. Standard deviation values are estimated to be below $2 \%$. Experimental error originates not only from the measurement of magnitude and phase of the scattering parameters, for which the uncertainties can be derived from the datasheet of the VNA, but also from the connectors mismatching and the distances between the reference planes and the sample. However, the dimensions of the containment area of the sample are well constrained, so that the experimental errors are minimized. It is estimated to be less than 10\% [Georget et al., 2014]. 


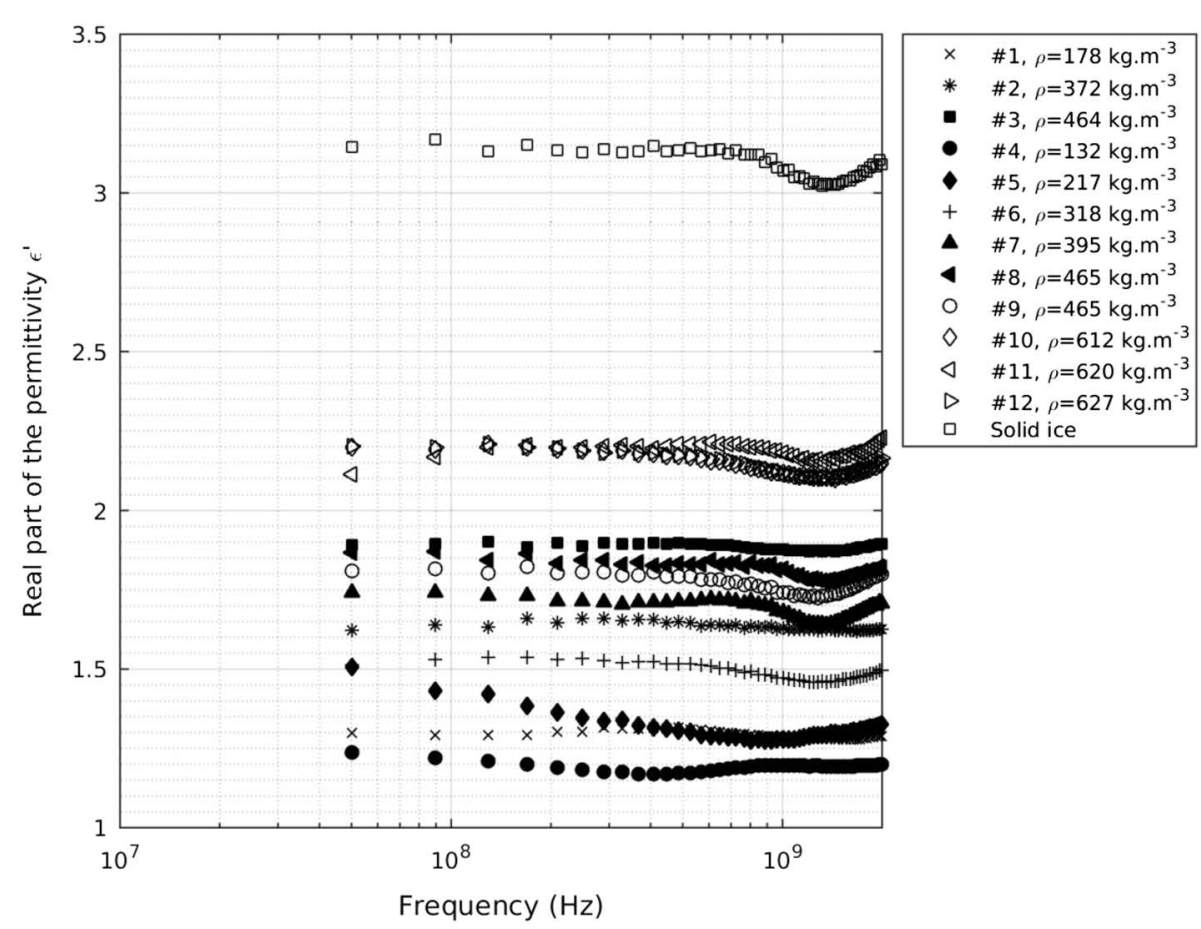

Figure 5. Mean of the real part of the permittivity as a function of the frequency for pure fine-grained ice samples (\#1-8), coarse-grained ice samples (\#9-12), and a solid ice sample (\#13). The mean values of $\epsilon^{\prime}$ are determined taking into account $\epsilon^{\prime}$ obtained from the scattering parameters $S_{11}$ and $S_{21}$, and $\epsilon^{\prime}$ obtained from the scattering parameters $S_{22}$ and $S_{12}$.

\section{Results}

\subsection{Frequency Dependence of Permittivity}

We determined the mean of $\epsilon^{\prime}$ for each frequency $v$, taking into account $\epsilon^{\prime}$ obtained from $S_{11}$ and $S_{21}$ (hereafter $\epsilon_{v}^{\prime}\left[S_{11}, S_{21}\right]$ ) and $\epsilon^{\prime}$ obtained from $S_{22}$ and $S_{12}$ (hereafter $\epsilon_{v}^{\prime}\left[S_{22}, S_{12}\right]$ ). Figure 5 shows the mean of the real part of the permittivity for the coarse-grained ice, fine-grained ice, and solid ice as a function of the measurement frequency on a logarithmic scale between $50 \mathrm{MHz}$ and $2 \mathrm{GHz}$. Figure 6 shows the mean $\epsilon^{\prime}$ for icy mixtures with a dust-to-ice volumetric ratio equal to $0.10,1.26$, and 2.83 , and Figure 7 shows the mean $\epsilon^{\prime}$ for icy mixtures with a dust-to-ice volumetric ratio equal to 0.31 , as a function of the measurement frequency on a logarithmic scale between $50 \mathrm{MHz}$ and $2 \mathrm{GHz}$. For all samples, the values of $\epsilon^{\prime}$ vary by less than $10 \%$ over the whole frequency range of measurement. These slight variations of $\epsilon^{\prime}$ are lower than the expected absolute errors of the measurements, meaning that they are not significative and that $\epsilon^{\prime}$ can be considered as nondispersive. This nondispersive behavior of $\epsilon^{\prime}$ is expected for this frequency range because of the nondispersive behavior of $\epsilon^{\prime}$ measured for the pure solid water ice [Mätzler and Wegmüller, 1987; Mattei et al., 2014] and JSC-1A lunar simulant [Brouet et al., 2015] at these frequencies. From a theoretical approach, it is also expected to get a nondispersive behavior at these frequencies for samples composed of water ice. Indeed, the single-relaxation Debye model, derived from the Kramers-Kronig relations, predicts well its dielectric behavior [Debye, 1929].

In order to study the homogeneity of each sample during the measurements, the maximum and minimum differences between $\epsilon_{v}^{\prime}\left[S_{11}, S_{21}\right]$ and $\epsilon_{v}^{\prime}\left[S_{22}, S_{12}\right]$, taking into account the whole range of frequency, are showed in Figure 8. The maximum difference varies between $1.5 \%$ for sample \#12 and $11 \%$ for sample \#39. Only 11 samples have a maximum difference larger than $5 \%$. However, for 10 samples, the number of frequencies for which the difference between $\epsilon_{v}^{\prime}\left[S_{11}, S_{21}\right]$ and $\epsilon_{v}^{\prime}\left[S_{22}, S_{12}\right]$ is larger than $5 \%$ represents only $10 \%$, at maximum, of the frequency band of measurement and $20 \%$ for the sample \#39. The good matching between $\epsilon_{v}^{\prime}\left[S_{11}, S_{21}\right]$ and $\epsilon_{v}^{\prime}\left[S_{22}, S_{12}\right]$ for all samples testifies of the homogeneity of the samples. Also, according to these results and their consistency with other measurements performed with different techniques [e.g., Mätzler, 1996], as discussed in section 5.1, the boundary effects, if any, are negligible. However, they should be carefully taken into account for measurements with samples composed of larger grains [Hickson et al., 2016]. 


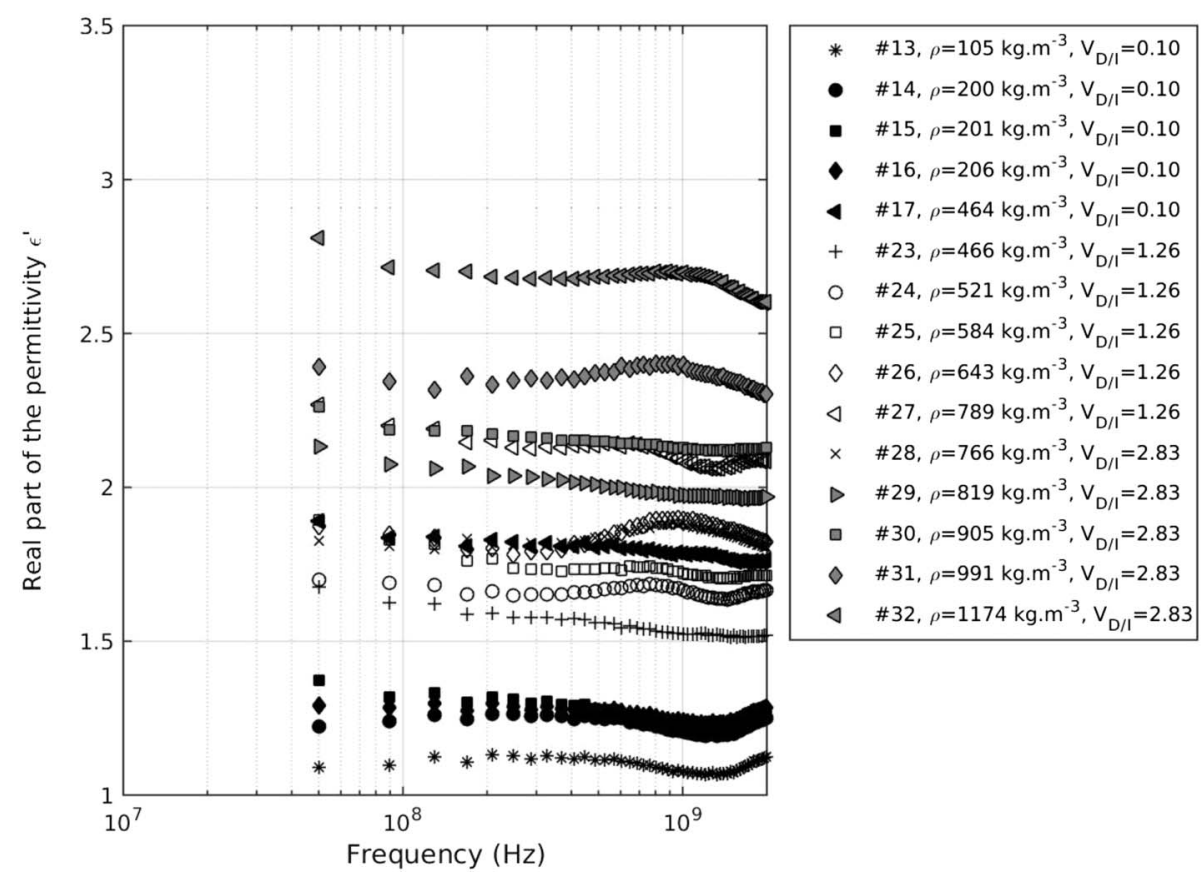

Figure 6. Mean of the real part of the permittivity as a function of the frequency for fine-grained mixtures with a dust-to-ice volumetric ratio equal to $0.10,1.26$, and 2.83. The mean values of $\epsilon^{\prime}$ are determined taking into account $\epsilon^{\prime}$ obtained from the scattering parameters $S_{11}$ and $S_{21}$, and $\epsilon^{\prime}$ obtained from the scattering parameters $S_{22}$ and $S_{12}$.

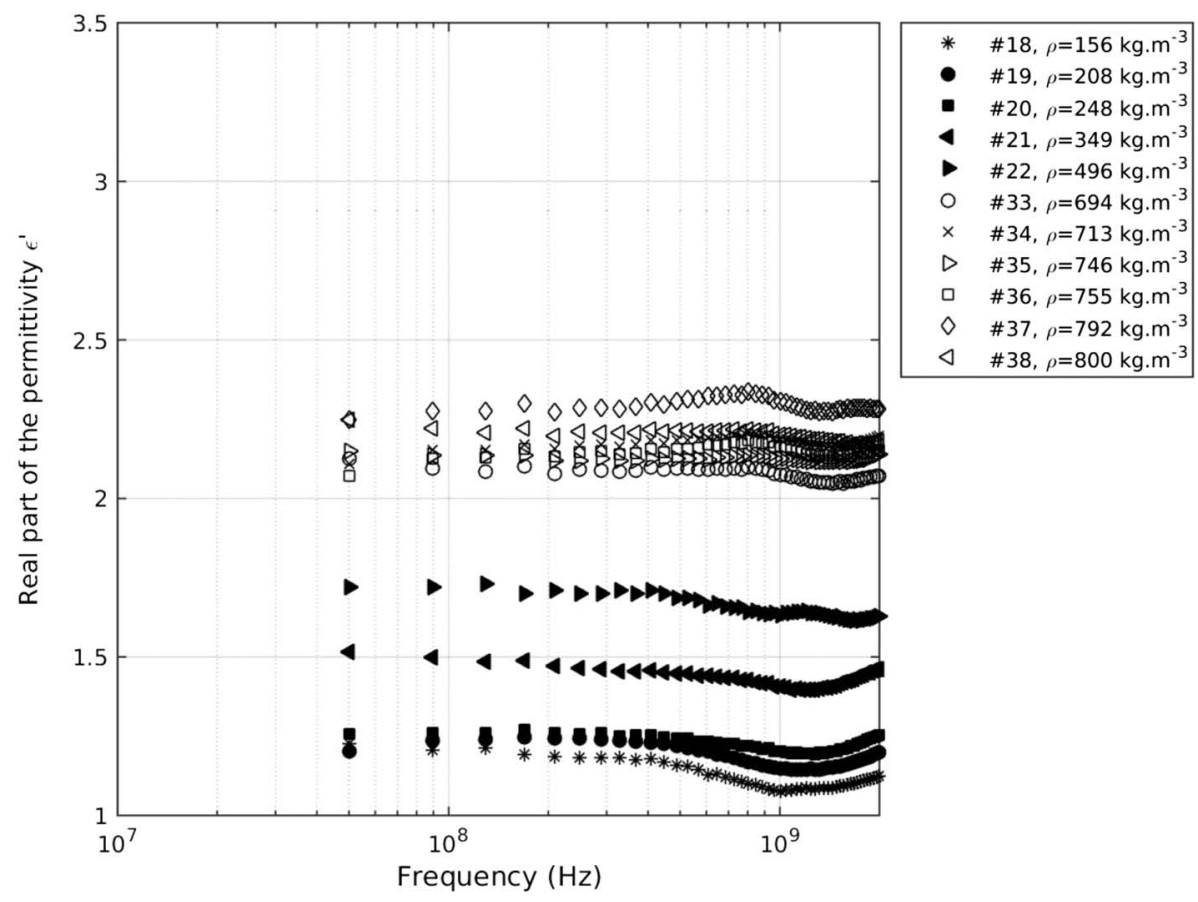

Figure 7. Mean of the real part of the permittivity as a function of the frequency for fine-grained mixtures (samples $\# 19-23$ ) and coarse-grained mixtures (samples \#34-39) with a dust-to-ice volumetric ratio equal to 0.31 . The mean values of $\epsilon^{\prime}$ are determined taking into account $\epsilon^{\prime}$ obtained from the scattering parameters $S_{11}$ and $S_{21}$, and $\epsilon^{\prime}$ obtained from the scattering parameters $S_{22}$ and $S_{12}$. 


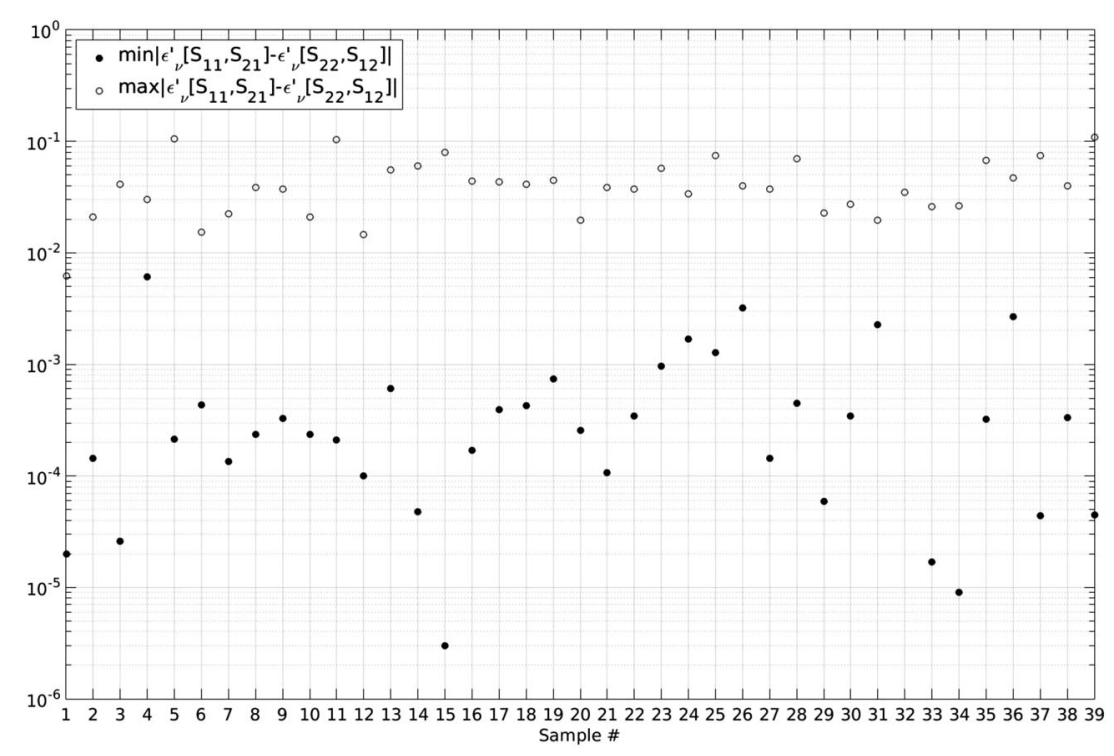

Figure 8. Maximum and minimum differences between $\epsilon^{\prime}$ obtained from $S_{11}$ and $S_{21}\left(\epsilon_{v}^{\prime}\left[S_{11}, S_{21}\right]\right)$ and $\epsilon^{\prime}$ obtained from $S_{22}$ and $S_{12}\left(\epsilon_{v}^{\prime}\left[S_{22}, S_{12}\right]\right)$ for each sample, taking into account the whole range of frequency. The homogeneity of the samples during the measurements is maintained, as showed by the low values of the differences.

\subsection{Bulk Density and Porosity Dependence of Permittivity}

In order to get a value of $\epsilon^{\prime}$ representative of a sample with a given bulk density or porosity, the mean of $\epsilon^{\prime}$ obtained from $S_{11}$ and $S_{21}$ and $\epsilon^{\prime}$ obtained from $S_{22}$ and $S_{12}$ is determined for each frequency. Then, from the nondispersive behavior of $\epsilon^{\prime}$ obtained for all samples, a mean value of $\epsilon^{\prime}$ is determined to be representative of $\epsilon^{\prime}$ over the whole frequency range. The mean values of $\epsilon^{\prime}$ for the fine-grained ice samples and the coarse-grained ice samples and the icy mixtures are expressed as a function of the bulk density in Figure 9 and the porosity in Figure 10. In Figure 9 the real part of the permittivity of the ice-dust mixtures appears to be lower than that for the pure ice samples for a given bulk density. If some particles of a pure water ice sample are replaced in the volume of the sample holder by basaltic dust with a similar grain size, with a solid density equal to 3 times the one of the ice, less water ice particles are needed to get the same bulk density and so the porosity of the sample is higher. Therefore, it is crucial to compare the results obtained on the icy mixtures and the pure ice samples as a function of the porosity in order to determine the effect of the dust content on the real part of the permittivity.

As expected, the real part of the permittivity increases with the increasing bulk density and decreases with the increasing porosity [e.g., Campbell and Ulrichs, 1969; Heggy et al., 2001; Ulaby and Long, 2014; Brouet et al., 2015]. Regarding the measurements done in October and in December on the fine-grained ice samples, the real part of the permittivity increases in the range of 1.2 to 1.9 with the increasing bulk density from 178 to $464 \mathrm{~kg} \mathrm{~m}^{-3}$ (i.e., a porosity in the range of 80.6 to $49.4 \%$ ) and from 132 to $465 \mathrm{~kg} \mathrm{~m}^{-3}$ (i.e., a porosity in the range of 85.6 to $49.3 \%$ ), respectively. For the coarse-grained ice samples, the real part of the permittivity is in the range of 1.7 to 2.2 with the increasing bulk density from 465 to $627 \mathrm{~kg} \mathrm{~m}^{-3}$ (i.e., a porosity in the range of 49.3 to $31.6 \%)$. The decrease of $\epsilon^{\prime}$ as a function of the increasing porosity, taking into account the measurements of the fine-grained ice samples, the coarse-grained ice samples, and the solid ice, is in fair agreement with values obtained from the Maxwell Garnett mixing law [Sihvola, 1999] for a mixture composed of air and solid water ice. For the icy mixtures, taking into account the different dust-to-ice volumetric ratios, the real part of the permittivity varies in the range of 1.1 to 2.7 for a porosity in the range of $90.6 \%$ to $42.1 \%$.

\subsection{Dust Content Dependence of Permittivity}

When the porosity is greater than $80 \%$, the values of $\epsilon^{\prime}$ are driven by the high content of air contained within the samples and the dust content does not have a significant effect. However, when the porosity is lower, the dust content has a measurable effect on $\epsilon^{\prime}$. The real part of the permittivity increases with the increasing dust content. For a porosity equal to about $75 \%, \epsilon^{\prime}$ is within the $1.4-1.7$ range for a dust-to-ice volumetric ratio lower than 3 , meaning a maximum variation of about $21 \%$. The lower the porosity, the stronger the effect of dust content on $\epsilon^{\prime}$. For a porosity of $50 \%, \epsilon^{\prime}$ increases from 1.8 for a pure grained ice sample to 2.1 


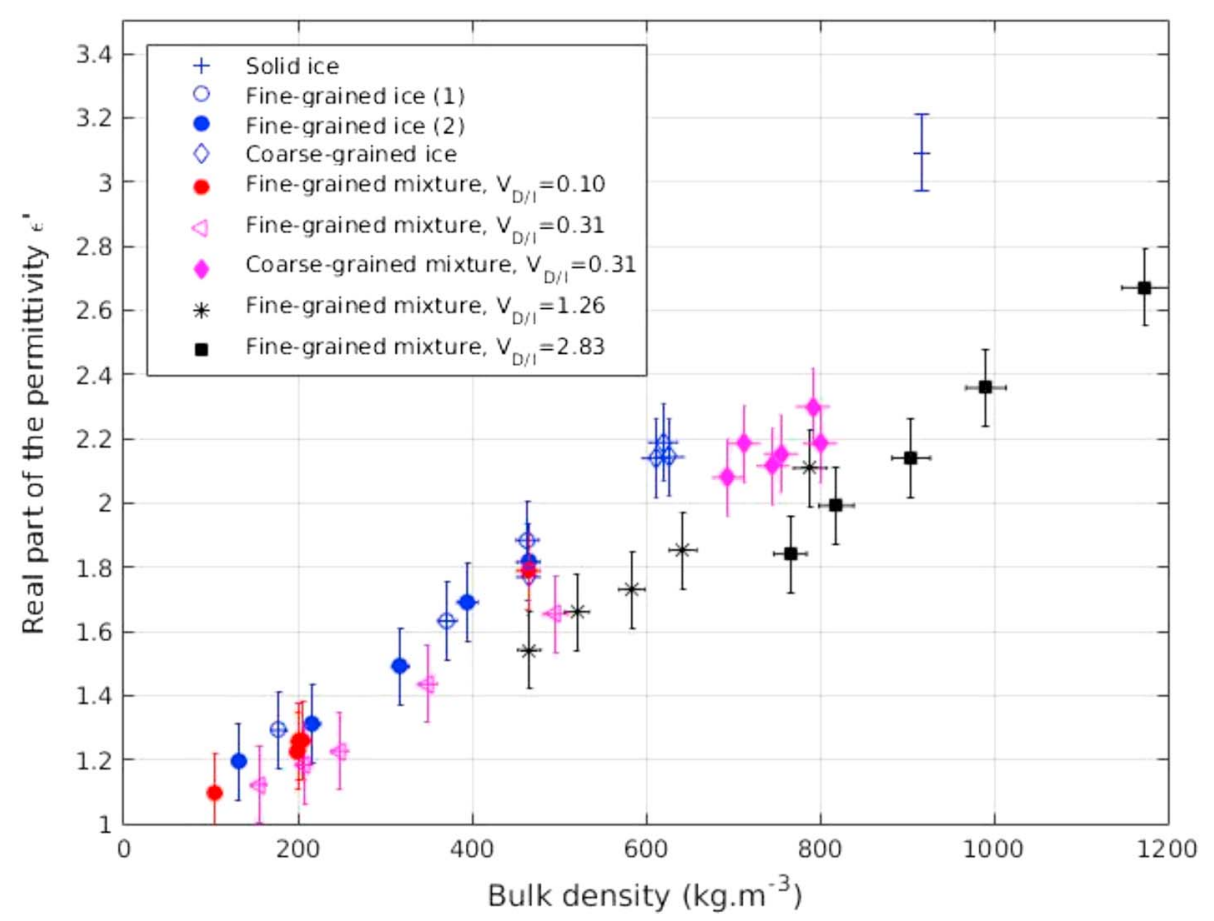

Figure 9. Mean real part of the permittivity, averaged over frequencies between $50 \mathrm{MHz}$ and $2 \mathrm{GHz}$, increasing with the increasing bulk density for pure water ices and ice-dust mixtures with different dust-to-ice volumetric ratio $V_{D / 1}$. The measurements on the fine-grained ice samples were performed in October (1) and in December 2015 (2). All other measurements were performed in December 2015. The error bars represent the sum of the maximum standard deviation over 10 acquisitions with the coaxial cell and the maximum experimental error.

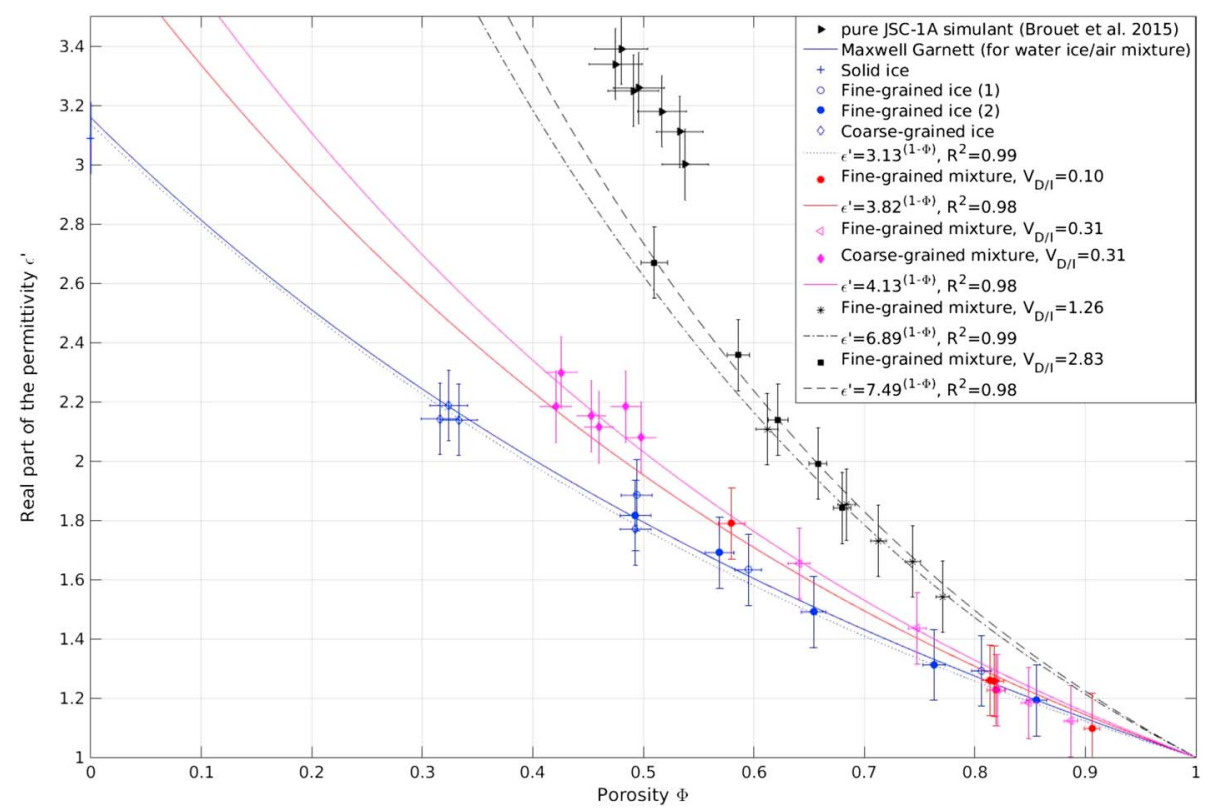

Figure 10. Mean real part of the permittivity, averaged over frequencies between $50 \mathrm{MHz}$ and $2 \mathrm{GHz}$, decreasing with the increasing porosity $\Phi$ for pure water ices and ice-dust mixtures with different dust-to-ice volumetric ratio $V_{D / /}$. The solid line represents $\epsilon^{\prime}$ as a function of $\Phi$ derived from the Maxwell Garnett mixing law applied for a two-component mixture (air and water ice). The fitted curves are obtained from the regression analyses applied to the data with a model based on equation (2). $R^{2}$ is the correlation coefficient, and the fitted coefficients are estimated with $95 \%$ of confidence bounds. The measurements on the fine-grained ice were performed in October (1) and in December 2015 (2). All other measurements were performed in December 2015. The error bars represent the sum of the maximum standard deviation over 10 acquisitions with the coaxial cell and the maximum experimental error. 
for an icy mixture with a dust-to-ice volumetric ratio equal to 0.31 (i.e., a dust mass fraction equal to 0.5 ) and is equal to about 2.7 for an icy mixture with a dust-to-ice volumetric ratio equal to 2.83 (i.e., a dust mass fraction equal to 0.9). Taking into account these values, $\epsilon^{\prime}$ increases by about $50 \%$ between a pure grained ice sample and an icy mixture with $V_{D / l}=2.83$ and by about $29 \%$ between icy mixtures with $V_{D / /}=0.31$ and $V_{D / l}=2.83$. In Figure 10, it is also important to remark that, as expected, the values of $\epsilon^{\prime}$ obtained for pure JSC-1A lunar simulant samples are larger than the ones obtained for the icy mixtures: for a porosity of about $52 \% \epsilon^{\prime}$ increases by about $20 \%$.

\subsection{Reproducibility of Measurements}

The methodology developed in this paper can be separated in two procedures, the sample preparation, as presented in section 2, and the measurement procedure, as presented in section 3 . By strictly following these procedures we were able to reproduce the bulk density and the porosity dependencies of the real part of the permittivity of the fine-grained ice measured in October 2015 with measurements taken 6 weeks later, as showed in Figure 10. We evaluated the reproducibility of the measurements by applying a linear regression analysis of the data obtained for fine-grained samples in October 2015 and those obtained in December 2015 as a function of the porosity. The results do not differ by more than $1 \%$. Thus, these measurements could be reproduced with pure ice or ice-dust mixtures with a different frequency range of measurement, temperature, and composition. This reproducibility is essential to make rigorous interpretation of observational data that have been or will be collected by GPR dedicated to icy subsurfaces [e.g., Kofman et al., 2010; Pettinelli et al., 2015].

\section{Discussion}

\subsection{Comparisons}

The results obtained by Mätzler [1996] from measurements in the microwave domain on dry snow collected in the Swiss and Austrian Alps are remarkably consistent with our results obtained on laboratory-grown fine-grained ice and coarse-grained ice. For instance, for a porosity equal to about 0.75 and $0.65, \epsilon^{\prime}$ is equal to about 1.4 and about 1.5 , respectively.

Hérique et al. [2002] have conducted permittivity measurements on ice-dust mixtures composed of water ice and powdered dunite, montmorillonite, and kaolinite, which have a silicate-rich composition. The measurements were performed at $120 \mathrm{~Hz}, 1.2 \mathrm{kHz}$, and $12 \mathrm{kHz}$ with temperatures in the range of 77 to $360 \mathrm{~K}$. The measured effective permittivity of the mixtures, dependent on the contribution of three components (i.e., dust, air, and ice), was greater than 3.9. Such values are consistent with the large contribution of the permittivity of the ice expected in this range of frequencies, where its relaxation spectrum is dependent on temperature. For a mixture composed of water ice and powdered dunite, which mostly consists of Mg-rich silicates as well as the JSC-1A simulant, the values were extrapolated to $90 \mathrm{MHz}$ and were found to be about 4.5 for a range of temperature of 150 to $250 \mathrm{~K}$ [Hérique et al., 2002]. These results are representative of materials with a very low porosity. The samples presented in this study have been prepared exerting mechanical pressure on the powder in a die. Our values obtained at $90 \mathrm{MHz}$ on ice-dust mixtures (also composed of water ice and silicate-rich dust) are lower because of the high content of air within the samples.

Permittivity measurements have been made by Mattei et al. [2014] on ice-dust mixtures with a composition similar to the samples presented in the current paper. Indeed, they have measured the permittivity of two mixtures composed of water ice and Etna volcanic ashes which have a basaltic composition, as well as the JSC-1 A lunar regolith simulant. The measurements were performed between $20 \mathrm{~Hz}$ and $1 \mathrm{MHz}$. The dust-to-ice volumetric ratio of the samples were 0.13 for a porosity equal to $5 \%$ and 0.96 for a porosity equal to $11 \%$. They obtained real parts of the permittivity around 3.5 and 5.0 at $1 \mathrm{MHz}$, respectively. Because the results suggest a nondispersive behavior of $\epsilon^{\prime}$ at frequencies above $500 \mathrm{kHz}$, one could compare these values with values obtained from models fitted to the data presented in Figure 10. For instance, for a dust-to-ice volumetric ratio of 0.10 and a porosity of $5 \%$, the model suggests a value of $\epsilon^{\prime}$ about $6 \%$ smaller than the value measured by Mattei et al. [2014]. Thus, the model predicts relatively well the measurement obtained by Mattei et al. [2014] for a very low porosity.

The heterogeneity of the permittivity measurements made by the authors mentioned above in terms of frequency, temperature, and composition ranges, as well as in terms of sample preparation technique, may also explain variations when comparing the results. 


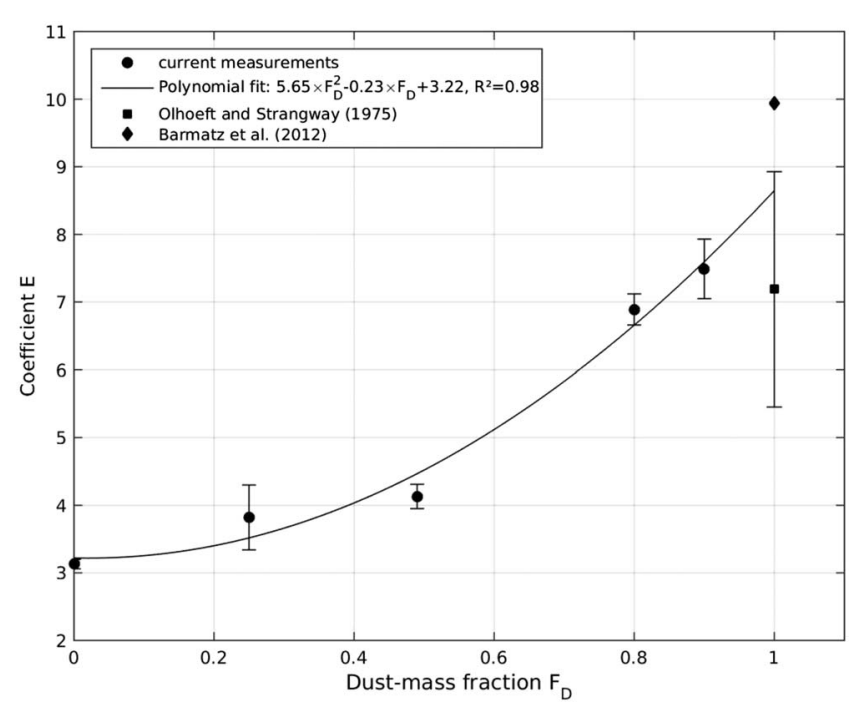

Figure 11. The fitted coefficient $E$ from equation 3 increases with the increasing dust mass fraction $F_{D}$. For $F_{D}=0$, the data obtained for samples $\# 1-12$ and solid ice (\#13) are taken into account in the regression analysis, samples \#14-18 for $F_{D}=0.25$, samples \#19-23 and \#34-39 for $F_{D}=0.49$, samples \#24-28 for $F_{D}=0.80$, and samples \#29-33 for $F_{D}=0.90 . R^{2}$ is the correlation coefficient, and the fitted coefficients of the polynomial function are estimated with $95 \%$ of confidence bounds.
5.2. Modeling the Porosity Dependence of $\epsilon^{\prime}$ as a Function of the Dust Content

Olhoeft and Strangway [1975] have reviewed measurements of the permittivity of 92 lunar soil samples at frequencies above $100 \mathrm{kHz}$ and determined a relation between the real part of the permittivity and the bulk density $\rho$ by fitting the data with a model based on the Lichtenecker formula:

$$
\log \left(\epsilon^{\prime}\right)=\sum_{k} V_{k} \log \left(\epsilon_{k}^{\prime}\right)
$$

with $\epsilon^{\prime}$ the real part of the permittivity of the mixture, $\epsilon_{k}^{\prime}$ and $V_{k}$ are the real part of the permittivity and the volume fraction of the $k$ th component, respectively. This formula can be reduced to $\epsilon^{\prime}=E^{\rho}$ for a two-component mixture composed of free space and lunar soil, with $E=\epsilon_{L}^{1 / \rho_{s}}$, with $\epsilon_{L}$ and $\rho_{s}$ the real part of the permittivity and the solid density of lunar soil, respectively.

The regression analysis of data reviewed by Olhoeft and Strangway [1975] gives the following relation $\epsilon^{\prime}=(1.93 \pm 0.17)^{\rho}$.

The current measurements presented in Figure 10 have also been fitted by a model derived from equation (2) to determine a relation between $\epsilon^{\prime}$ and the porosity for pure water ice, on the one hand, and icy mixtures, on the other hand. The model can be reduced to

$$
\epsilon^{\prime}=E^{(1-\Phi)}
$$

with $E=\left(\frac{\epsilon_{D}^{\prime}}{\epsilon_{l}^{\prime}}\right)^{V_{D}} \times \epsilon_{l}^{\prime}$, the fitted parameter for the pure ice data (with a volume fraction of dust $V_{D}$ equal to 0 ) and icy mixture data where $\epsilon_{D}^{\prime}$ and $\epsilon_{l}^{\prime}$ are, respectively, the real part of the permittivity of the pure dust and the pure solid water ice. Results of all regression analyses are summarized in Figure 11 which shows that the coefficient $E$ from equation (3) increases with the increasing dust mass fraction. From $F_{D}=0$ to $F_{D}=0.90, E$ increases from $3.13 \pm 0.06$ to $7.49 \pm 0.44$. The fitted parameter for pure ice data, which is the real part of the permittivity of solid water ice, is consistent with the value given by Gough [1972] and Mätzler and Wegmüller [1987] for temperatures equal to or greater than $200 \mathrm{~K}$.

The predicted value for a pure sample of JSC-1A simulant, obtained from a regression analysis of $E$ as a function of the dust mass fraction, is consistent with the value of Olhoeft and Strangway [1975] obtained from lunar soil samples and Barmatz et al. [2012] obtained from lunar simulants and some lunar soil samples. Based on the experimental data, we propose an empirical model to determine the real part of the permittivity as a function of the porosity $\Phi$ and the dust mass fraction $F_{D}$ :

$$
\epsilon^{\prime}\left(F_{D}, \Phi\right)=\left(5.65 \times F_{D}^{2}-0.23 \times F_{D}+3.22\right)^{1-\Phi} .
$$

This model could be a support for data inversion of GPRs operating between $50 \mathrm{MHz}$ and $2 \mathrm{GHz}$ dedicated to the investigation of icy planetary subsurfaces and the internal structure of porous and icy bodies, such as cometary nuclei. Moreover, the nondispersive behavior of the real part of the permittivity of the samples suggests that this model could be applied to a broader frequency range from about $10 \mathrm{MHz}$ to hundreds of $\mathrm{GHz}$. Also, the temperature dependence of $\epsilon^{\prime}$ has to be taken into account. The temperature dependence of $\epsilon^{\prime}$ has been estimated to be linear for solid water ice between $10 \mathrm{MHz}$ and $300 \mathrm{GHz}$ [Mätzler and Wegmüller, 1987] and for the JSC-1A lunar simulant [Calla and Rathore, 2012] at $1.7 \mathrm{GHz}$. These measurements show that the slope of variation of $\epsilon^{\prime}$ with the temperature is of the order of $10^{-3}$ in the frequency range of our measurements. 


\subsection{Relevance for the Sounding of Icy Media in the Solar System With Radio Waves}

In order to interpret the GPRs data, parameters related to the propagation of the transmitted wave have to be estimated in a first order, such as the phase velocity of the radio wave and the attenuation constant of the medium. Both depend on the product of the real part of the permittivity with the magnetic permeability of the medium [e.g., Ulaby and Long, 2014]. However, as water ice, the JSC-1A lunar simulant can be considered as nonmagnetic from the measurements performed by Barmatz et al. [2012], meaning that its magnetic permeability is equal to that of free space. Then the phase velocity $v_{p}$ of the radio wave which would propagate in icy and/or porous media, similar to the nonmagnetic materials reported in this study, can be expressed by $v_{p}=c / \sqrt{\epsilon^{\prime}}$, with $c$ the speed of light in a vacuum, and the attenuation constant $\alpha\left(\mathrm{Np} \mathrm{m}^{-1}\right)$ for one-way propagation as follows [Ulaby and Long, 2014]:

$$
\alpha=\frac{2 \pi}{\lambda_{0}}\left(\frac{\epsilon^{\prime}}{2}\left[\sqrt{1+\frac{\epsilon^{\prime \prime}}{\epsilon^{\prime}}}-1\right]\right)^{1 / 2}
$$

where $\lambda_{0}$ is the wavelength in free space. The attenuation constant depends on the real part of the permittivity, as well as on the imaginary part. As mentioned in section 3 , the sensitivity of the instrument used for the permittivity measurements does not allow to properly measure the imaginary part of the samples. However, it is possible to discuss about the upper limits of $\epsilon^{\prime \prime}$ for the two poles of the mixtures, i.e., water ice and the JSC-1 A simulant. For solid water ice, $\epsilon^{\prime \prime}$ has been measured below 0.001 in the frequency range of the measurements [Mätzler and Wegmüller, 1987]. Then for an increasing porosity, $\epsilon^{\prime \prime}$ may decrease of a few orders of magnitude [Heggy et al., 2012]. Regarding the JSC-1A simulant, Calla and Rathore [2012] have measured $\epsilon^{\prime \prime}$ at $1.7 \mathrm{GHz}$ in the range of 0.15 to 0.43 for a temperature range of about $80 \mathrm{~K}$ to $470 \mathrm{~K}$ and for a porosity equal to $38 \%$. At $300 \mathrm{~K}, \epsilon^{\prime \prime}$ is equal to 0.29 . In addition, these measurements allow to derive a linear temperature dependence of $\epsilon^{\prime \prime}$ with a slope equal to about $6.5 \times 10^{-4} \mathrm{~K}^{-1}$, which can be used to estimate $\epsilon^{\prime \prime}$ in a low-temperature environment. Barmatz et al. [2012] have also measured $\epsilon^{\prime \prime}$ of the JSC-1A simulant at room temperature. For a porosity equal to $43 \%, \epsilon^{\prime \prime}$ is in the range of 0.055 to 0.073 . Thus, for larger porosities, the upper limit of $\epsilon^{\prime \prime}$ would be equal to 0.07 for temperatures below $300 \mathrm{~K}$.

\subsubsection{Relevance for Cometary Nuclei, Especially 67P/Churyumov-Gerasimenko}

The high values of porosity of our samples, i.e., between $50 \%$ and $91 \%$, are representative of porosities expected for cometary nuclei [Lamy et al., 2015]. More particularly, this range of porosity encompasses the values of porosity estimated for the nucleus 67P, which has been targetted by the Rosetta mission. Moreover, the samples consist of pure water ice and refractory dust, which can be considered, in a first approach, as the two main phases of cometary material [e.g., Festou et al., 2004; Lamy et al., 2015]. The large range of dust mass fraction of the samples also allows to simulate the properties of an icy nucleus or a dusty nucleus. Thus, the results presented in this paper are, first, relevant for the interpretation of the measurements of the electrical properties of the upper part of the small lobe of 67P by the CONSERT penetrating radar, operating at $90 \mathrm{MHz}$. The CONSERT bistatic measurements between the Rosetta orbiter and the Philae lander were performed during the First Science Sequence. In order to derive a value of $\epsilon^{\prime}$ representative of the medium crossed by the radio waves, a range of a priori values have been tested by Kofman et al. [2015] in a model of signal propagation between Philae and Rosetta, taking into account the three-dimensional shape model of the nucleus, aiming to reproduce the data measured by CONSERT. For the porosity range estimated for 67P by Sierks et al. [2015] and Pätzold et al. [2016], i.e., in the range of 70\% to $80 \%$, equation (4) predicts a value of $\epsilon^{\prime}$ in the $1.2-1.9$ range for a dust fraction in the range of 0 to 1 . Taking into account the temperature dependence, the values predicted with our model for temperatures below $200 \mathrm{~K}$ validate the range of $\epsilon^{\prime}$ considered for the CONSERT simulations (from 1.025 to 1.45) and encompass the value of $\epsilon^{\prime}$ derived by Kofman et al. [2015], which is equal to $1.27 \pm 0.05$. Furthermore, from data collected by the permittivity probe (hereafter PP) of the SESAME package (Surface Electric Sounding and Acoustic Monitoring Experiment) [Seidensticker et al., 2007] during the FSS, Lethuillier et al. [2016] have determined a lower limit of $\epsilon^{\prime}$ equal to 2.45 for the near surface of the small lobe, at the final landing site of Philae, in the $400-800 \mathrm{~Hz}$ range of frequency. Based on the results presented in the current paper and the results obtained by the CONSERT and SESAME-PP instruments, and taking into account the temperature conditions during the FSS, Brouet et al. [2016] show that the porosity increases with depth in the small lobe of the nucleus.

The nondispersive behavior of all mixture samples and of each component considered separately in the frequency range of $50 \mathrm{MHz}$ to $2 \mathrm{GHz}$ implies that the results of the measurements presented in this paper and the 
equation (4) can be used to estimate the real part of the permittivity of porous materials sounded at higher frequencies, taking into account the temperature dependence of $\epsilon^{\prime}$ estimated in section 5.2. The Microwave Instrument for the Rosetta Orbiter (MIRO) [Gulkis et al., 2007] on board the Rosetta spacecraft is a radiometer composed of a millimeter receiver with a center-band frequency equal to $190 \mathrm{GHz}$ (i.e., a wavelength equal to $1.6 \mathrm{~mm}$ in the vacuum) and a submillimeter receiver with a center-band frequency equal to $560 \mathrm{GHz}$ (i.e., a wavelength equal to $0.5 \mathrm{~mm}$ in the vacuum). MIRO has performed measurements of the near-surface brightness temperatures and temperature gradients in the uppermost layers of 67P [Gulkis et al., 2015]. The brightness temperatures observed depend on the penetration depth for each of the MIRO wavelengths, which is related to the thermal and physical properties of the material via its permittivity. Thus, the permittivity is a key parameter of inverse models used to interpret data of brightness temperatures [e.g., Schloerb et al., 2015; Choukroun et al., 2015]. Our results provide constraints on the real part of the permittivity of porous and icy near surface of 67P and on its porosity range, as well as dust fraction.

\subsubsection{Relevance for Planetary Objects}

The large porosity range of our measurements makes them relevant not only for the investigations of the internal properties of highly porous objects but also for the investigation with GPRs of the crustal properties of icy planetary objects. Results presented for porosities below $50 \%$ may be taken into account in the preparation of the interpretation of GPRs data. Actually, the interior of Jupiter's icy moons are planned to be investigated by GPRs. The European JUICE (JUpiter ICy moons Explorer) [Grasset et al., 2013] spacecraft, scheduled to be launched in 2022, will carry two radiometric experiments. The Submillimeter Wave Instrument (SWI) will measure brightness temperatures of near surfaces of Jupiter's icy moons in two spectral ranges: $530-601 \mathrm{GHz}$ and 1080-1275 GHz. However, our results may not be relevant for frequencies larger than $900 \mathrm{GHz}$. Indeed, Bertie et al.'s [1969] measurements of water ice in the $900-1600 \mathrm{GHz}$ range seem to exhibit a relaxation process, for which the effect still needs to be constrained in laboratory for water ice-dust mixtures. The JUICE mission foresees to understand the icy crustal structure of Ganymede, Callisto, and Europa with the RIME (Radar for Icy Moon Exploration) [Bruzzone et al., 2013] penetrating radar, which operates at a center-band frequency of $9 \mathrm{MHz}$. Moreover, selected to be part of the NASA's planned Europa mission [Pappalardo et al., 2015], the REASON (Radar for icy Moon Exploration) [Grima et al., 2013] penetrating radar will precisely focus on the determination of the internal structure of the Jupiter's icy moon Europa. Earth-based spectroscopic observations have showed that the surface of the three Galilean moons is mostly composed of water ice [e.g., Johnson and McCord, 1971; Pilcher et al., 1972; Fink et al., 1973]. The measured surface temperatures vary in the range of $70 \mathrm{~K}$ to $200 \mathrm{~K}$ [e.g., Pettinelli et al., 2015; Ligier et al., 2016]. According to the solid phase diagram of the water ice [Bartels-Rausch et al., 2012], the surface conditions imply that the water ice is present in its Ih crystalline form [Ligier et al., 2016]. As pressure increases with depth, ice lh could transform to other phases in depth. Nevertheless, these phase transitions should occur at depths larger than those expected to be sounded by the RIME and REASON radars, estimated to be in the range of a few kilometers to $10 \mathrm{~km}$ [Pettinelli et al., 2015]. The presence of amorphous water ice is also inferred on the surface of the Jovian satellites [Hansen and McCord, 2004; Dalton et al., 2010]: it is predominant on Europa's surface, in smaller proportion on Callisto's surface, and both phases are present on Ganymede's surface. However, Hansen and McCord [2004] suggest that the crystalline phase is predominant at a depth around $1 \mathrm{~mm}$ for the three satellites. The water Ih ice particles used in the current measurements have been prepared with a controlled technique, which enables their size distribution and their quasi-spherical shape to be defined. Knowing their properties, they can be considered representative of the water ice particles which constitute the icy crusts of the three Jupiter's moons. Indeed, at least for Europa, the size distribution of the ice particles has been estimated to be in the range of $25 \mu \mathrm{m}$ to $200 \mu \mathrm{m}$, based on infrared spectra of the surface obtained from Earth-based observations [Ligier et al., 2016]. In order to characterize the structure of the icy crusts, their dielectric properties have to be estimated taking into account the possible contaminants mixed within the water ice. The procedures of sample preparation applied for the pure ice samples, as well as for mixture samples, described in section 2, allow us to control the porosity and the dust mass fraction of the samples. These procedures and the measurement procedure described in section 3, providing reproducible results around $243 \mathrm{~K}$, could serve for the preparation of a database to support the preparation of the interpretation of RIME and REASON data, taking into account mixtures composed of fine-grained water ice and contaminants identified at the surface the Galilean icy moons, such as $\mathrm{CO}_{2}$ ice, hydrated salt minerals, sulfur-bearing species, or organic compounds [e.g., McCord et al., 1998; Grasset et al., 2013]. Measurements performed by Pettinelli et al. [2016] on ice/ $\mathrm{MgSO}_{4} \cdot 11 \mathrm{H}_{2} \mathrm{O}$ mixtures in the frequency range of $100 \mathrm{~Hz}$ to $1 \mathrm{MHz}$ and over a temperature range of $100 \mathrm{~K}$ to $260 \mathrm{~K}$ are also relevant for this particular purpose. Constraining the composition and the internal structure of the icy crusts 
will help to identify the liquid water reservoirs beneath the icy crusts of Ganymede, Europa, and Callisto from GPRs data [e.g., Spohn and Schubert, 2003], which have been proposed from geophysical models based on Galileo observations [Zimmer et al., 2000; Grasset et al., 2013].

In addition, the measurements reported in this study may be taken into account in the interpretation of the data collected on Martian polar ice caps, which have been investigated by two subsurface sounding radars: MARSIS (Mars Advanced Radar for Subsurface and lonosphere Sounding), operating in the frequency range of 1.3 to $5.5 \mathrm{MHz}$ [Jordan et al., 2009]; and SHARAD (Shallow Radar), operating in the range of $10 \mathrm{MHz}$ to $30 \mathrm{MHz}$ [Seu et al., 2004]. They are carried respectively on ESA's Mars Express and NASA's Mars Reconnaissance Orbiter. Our results may help to affine the data inversion as a function of the porosity and dust fraction of the different geological units encountered in the subsurface. Moreover, in 2020, the European Space Agency will launch the ExoMars rover equipped with the WISDOM penetrating radar (Water Ice Subsurface Deposit Observation on Mars) [Ciarlettiet al., 2012] operating in the frequency range of $500 \mathrm{MHz}$ to $3 \mathrm{GHz}$ and dedicated to perform large-scale investigations of the Martian shallow subsurface at the rover location. WISDOM will aim to investigate and characterize the nature of the subsurface and subsurface stratigraphy, structure, and the magnitude and scale of spatial heterogeneity.

\section{Conclusions}

We report permittivity measurements of 12 porous water ice samples and 26 samples of water ice-dust mixtures, with the JSC-1A lunar regolith simulant as the dust component, in the frequency range of $50 \mathrm{MHz}$ to $2 \mathrm{GHz}$ at a temperature around $243 \mathrm{~K}$. The procedures of sample preparation and the method of permittivity measurement were controlled in order to ensure the reproducibility of the measurements. The porosity of the samples ranges from about 31\% to $91 \%$. The real part of the permittivity of the samples is accurately measured in the range of 1.1 to 2.7. A nondispersive behavior has been observed for all samples. These results contribute to the knowledge on the porosity dependence of laboratory-grown water ice and water ice-dust mixtures as a function of the dust content.

From these experimental data, models are obtained and suggest that $\epsilon^{\prime}$ varies with the porosity according to $E^{(1-\Phi)}$ with $E=3.13,3.82,4.13,6.89$, and 7.49 for pure water ice and for ice-dust mixtures with a dust-to-ice volumetric ratio equal to $0.10,0.31,1.26$, and 2.83 , respectively. Moreover, from these analyses, a model encompassing the dust content and the porosity dependencies is proposed. These results are reliable in the frequency range of $50 \mathrm{MHz}$ to $2 \mathrm{GHz}$. Also, they could be reliable for lower frequencies, to about $10 \mathrm{MHz}$, as well as for larger frequencies because of the nondispersive behavior of the mixtures and also of the pure water ice and the pure JSC-1 A lunar simulant at these frequencies. Nevertheless, confirmation from laboratory measurements on icy mixtures has still to be obtained in the millimeter-submillimeter domains. These data and models are relevant for data inversion of GPRs dedicated to the internal investigation of porous media in the solar system, such as rubble pile asteroids, transneptunian objects, and cometary nuclei, and more especially the nucleus of comet 67P, which was sounded by the CONSERT radar onboard the Rosetta spacecraft in November 2014. The permittivity measurements of such porous samples are complex because of the preparation of the samples themselves and their low-loss properties. Future laboratory measurements dedicated to the investigation of the permittivity (real and imaginary parts) of different icy mixtures with impurities, such as salts, analogues of extraterrestrial organic matter (e.g., tholins), and powdered meteorites, could be conducted using the same procedures of sample preparation.

In addition, these developments could have implications for the data inversion of MARSIS or SHARAD experiments for the study of the Martian polar subsurface and in the near future, the WISDOM (Water Ice Subsurface Deposit Observation on Mars) GPR, which has been selected by the European Space Agency to be carried by the ExoMars Rover, and will sound the near subsurface of Mars with a frequency range from 0.5 to $3 \mathrm{GHz}$. The missions toward icy satellites with launches scheduled in the next decade, such as the JUpiter ICy moons Explorer (JUICE) mission and NASA's Europa mission will also carry GPRs.

Acknowledgments

Support from the Swiss National

Science Foundation is acknowledged.

We also acknowledge the Centre

National d'Etude Spatiale (CNES,

France) for its support. Data used

for this study can be accessed

from Y.B. (Yann Brouet; e-mail:

yann.brouet@space.unibe.ch).

\section{References}

Alshibli, K. A., and A. Hasan (2009), Strength properties of JSC-1A lunar regolith simulant, J. Geotec. Geoenviron., 135, 673-679.

Barmatz, M., et al. (2012), Microwave permittivity and permeability measurements on lunar simulants, in 43th Lun. Planet. Sci. Conf., vol. 1659, p. 1050, LPI Contribution, Woodlands, Tex.

Bartels-Rausch, T., et al. (2012), Ice structures, patterns, and processes: A view across the icefields, Rev. of Mod. Phys., 84, 885-944.

Barucci, M. A., H. Boehnhardt, D. P. Cruikshank, and A. Morbidelli (2008), The Solar System Beyond Neptune, Tucson, Ariz. 
Barucci, M. A., E. Dotto, and A. C. Levasseur-Regourd (2011), Space missions to small bodies: Asteroids and cometary nuclei, Astron. Astrophys. Rev., 19, 48.

Bertie, J. E., H. J. Labbe, and E. Whalley (1969), Absorptivity of ice I in the range $4000-30 \mathrm{~cm}^{-1}$, J. Chem. Phys., 50, 4501-4520.

Brouet, Y., A. C. Levasseur-Regourd, P. Encrenaz, and S. Gulkis (2014), Permittivity of porous granular matter, in relation with Rosetta cometary mission, Planet. Space Sci., 103, 143-152.

Brouet, Y., et al. (2015), Permittivity measurements of porous matter in support of investigations of the surface and interior of 67P/Churyumov-Gerasimenko, Astron. Astrophys., 583, A39.

Brouet, Y., et al. (2016), A porosity gradient in 67P/C-G nucleus suggested from CONSERT and SESAME-PP results: An interpretation based on new laboratory permittivity measurements of porous icy analogues, Mon. Not. R. Astron. Soc., 462, 89-98.

Bruzzone, L., et al. (2013), RIME: Radar for Icy Moon Exploration, in International on Geoscience and Remote Sensing Symposium (IGARSS), pp. 3907-3910, IEEE, London.

Calla, O. P. N., and I. S. Rathore (2012), Study of complex dielectric properties of lunar simulants and comparison with Apollo samples at microwave frequencies, Adv. Space Res, 50, 1607-1614.

Campbell, M. J., and J. Ulrichs (1969), Electrical properties of rocks and their significance for lunar radar observations, J. Geophys. Res, 74, 5867-5881.

Cartwright, J. H. E. (2007), Ice in the solar system, in Astronomical Society of the Pacific Conference Series, vol. 370, edited by S. O. Demircan and B. Albayrak, p. 265, Astron. Soc. of the Pacific, San Francisco, Calif.

Choukroun, M., et al. (2015), Dark side of comet 67P/Churyumov-Gerasimenko in August-October 2014. MIRO/Rosetta continuum observations of polar night in the southern regions, Astron. Astrophys., 583, A28.

Ciarletti, V., et al. (2012), WISDOM a GPR for the ExoMars rover mission, in International Workshop on Instrumentation for Planetary Missions, vol. 1683, p. 1126, LPI Contributions. [Available at http://www.lpi.usra.edu/meetings/ipm2012/pdf/1126.pdf.]

Ciarletti, V., A. C. Levasseur-Regourd, J. Lasue, C. Statz, D. Plettemeier, A. Hérique, Y. Rogez, and W. Kofman, (2015), CONSERT suggests a change in local properties of 67P/Churyumov-Gerasimenko's nucleus at depth, Astron. Astrophys., 583, A40, doi:10.1051/0004-6361/201526337.

Cole, K. S., and R. H. Cole (1941), Dispersion and absorption in dielectrics. I. Alternating current characteristics, J. Chem. Phys., 9(4), 341-351. Dalton, J. B., et al. (2010), Chemical composition of icy satellite surfaces, Space Sci. Rev., 153, 113-154.

Debye, P. (1929), Polar molecules., J. Soc. Chem. Ind., 48, 1036-1037.

Festou, M. C., H. U. Keller, and H. A. Weaver (2004), Comets II, Univ. Arizona Press, Tucson, Ariz.

Fink, U., N. H. Dekkers, and H. P. Larson (1973), Infrared spectra of the Galilean satellites of Jupiter, Astrophys. J., 179, 155-159, doi:10.1086/181139.

Fulle, M., et al. (2000), In situ dust measurements from within the Coma of 1P/Halley: First-order approximation with a dust dynamical model, Astron. J., 119, 1968-1977.

Fulle, M., et al. (2016), Comet 67P/Churyumov-Gerasimenko preserved the pebbles that formed planetesimals, Mon. Not. R. Astron. Soc., 462, S132-S137, doi:10.1093/mnras/stw2299.

Georget, E., R. Abdeddaim, and P. Sabouroux (2014), A quasi-universal method to measure the electromagnetic characteristics of usual materials in the microwave range, C. R. Phys., 15, 448-457.

Gough, S. R (1972), A low temperature dielectric cell and the permittivity of hexagonal ice to 2 K, Can. J. Chem., 50, 3046-3051.

Grasset, O., et al. (2013), JUpiter ICy moons Explorer (JUICE): An ESA mission to orbit Ganymede and to characterise the Jupiter system, Planet. Space Sci., 78, 1-21.

Gulkis, S., et al. (2007), MIRO: Microwave Instrument for Rosetta Orbiter, Space Sci. Rev., 128, 561-597.

Gulkis, S., et al. (2015), Subsurface properties and early activity of comet 67P/Churyumov-Gerasimenko, Science, 347, aaa0709, doi:10.1126/science.aaa0709.

Grima, C., D. M. Schroeder, and D. D. Blankenship (2013), Identifying surface characteristics with an ice penetrating radar sounder at Europa: Potential for landing site selection, in 44th Lunar and Planetary Science Conference, vol. 1719, p. 2980, LPI Contribution, Woodlands, Tex.

Hansen, G. B, and T. B. McCord (2004), Amorphous and crystalline ice on the Galilean satellites: A balance between thermal and radiolytic processes, J. Geophys. Res., 109, E1012, doi:10.1029/2003JE002149.

Heggy, E., et al. (2001), On water detection in the Martian subsurface using sounding radar, Icarus, 154, 244-257.

Heggy, E., et al. (2012), Radar properties of comets: Parametric dielectric modeling of Comet 67P/Churyumov-Gerasimenko, Icarus, 221, 925-939.

Hérique, A., J. Gilchrist, W. Kofman, and J. Klinger (2002), Dielectric properties of comet analog refractory materials, Planet. Space Sci., 50 , 857-863.

Hickson, D., S. Sotodeh, M. G. Daly, R. Ghent, and M. C. Nolan (2016), Improvements on effective permittivity measurements of powdered alumina: Implications for bulk permittivity properties of asteroid regoliths, Adv. Space Res., doi:10.1016/j.asr.2016.08.011, in press.

Hill, E., M. J. Mellin, B. Deane, Y. Liu, and L. A. Taylor (2007), Apollo sample 70051 and high- and low-Ti lunar soil simulants MLS-1A and JSC-1 A: Implications for future lunar exploration, J. Geophys. Res., 112, E02006, doi:10.1029/2006JE002767.

Johari, G. P., and E. Whalley (1981), The dielectric properties of ice Ih in the range 272-133 K, J. Chem. Phys., 75, 1333-1340.

Johnson, T. V, and T. B. McCord (1971), Spectral geometric albedo of the Galilean satellites, 0.3 to 2.5 microns, Astrophys. J., 169, 589-594.

Jordan, R., et al. (2009), The Mars express MARSIS sounder instrument, Planet. Space Sci., 57, 1975-1986.

Jost, B., et al. (2016), Experimental characterization of the opposition surge in fine-grained water-ice and high albedo ice analogs, Icarus, 264, 109-131.

Kofman, W., et al. (1998), Comet nucleus sounding experiment by radiowave transmission, Adv. Space Res., 21, 1589-1598.

Kofman, W., R. Orosei, and E. Pettinelli (2010), Radar signal propagation and detection through ice, Space Sci. Rev., 153, 249-271.

Kofman, W., et al. (2015), Properties of the 67P/Churyumov-Gerasimenko interior revealed by CONSERT radar, Science, 349, aab0639, doi:10.1126/science.aab0639.

Lamy, P. L., et al. (2015), The subsurface structure and density of cometary nuclei, Space. Sci. Rev., 197, 85-99, doi:10.1007/s11214-015-0160-0.

Lethuillier, A., et al. (2016), Electrical properties and porosity of the first meter of the nucleus of 67P/Churyumov-Gerasimenko. As constrained by the permittivity probe SESAME-PP/Philae/Rosetta, Astron. Astrophys., 591 , A32.

Levasseur-Regourd, A. C., E. Hadamcik, E. Desvoivres, and J. Lasue (2009), Probing the internal structure of the nuclei of comets, Planet. Space Sci., 57, 221-228.

Lide, A. H. (2005), Handbook of Chemistry and Physics, CRC Press, Florida.

Ligier, N., et al. (2016), VLT/SINFONI observations of Europa: New insights into the surface composition, Astron. J., 151(6), 163, doi:10.3847/0004-6256/151/6/163. 
Mattei, E., et al. (2014), Dielectric measurements and radar attenuation estimation of ice/basalt sand mixtures as Martian polar caps analogues, Icarus, 229, 428-433.

Mätzler, C., and U. Wegmüller (1987), Dielectric properties of fresh-water ice at microwave frequencies, J. Phys. D: App. Phys., 20, $1623-1630$.

Mätzler, C. (1996), Microwave permittivity of dry snow, IEEE Trans. Geosci. Remote Sens., 34, 573-581.

Mätzler, C., W. Ellison, B. Thomas, A. Sihvola, and M. Schwank (2006), Dielectric properties of natural media, in IET Electromagnetic Waves Series, vol. 52, edited by C. Mätzler, pp. 427-505, London.

McCord, T. B., et al. (1998), Non-water-ice constituents in the surface material of the icy Galilean satellites from the Galileo near-infrared mapping spectrometer investigation, J. Geophys. Res., 103, 8603-8626.

Nicolson, A. M., and G. F. Ross (1970), Measurement of the intrinsic properties of materials by time-domain techniques, IEEE Trans. Instr. and Meas., 19, 377-382.

Olhoeft, G. R., and D. W. Strangway (1975), Dielectric properties of the first 100 meters of the Moon, Earth Planet. Sc. Lett., $24,394-404$.

Palmer, E. M., E. Heggy, M. T. Capria, and F. Tosi (2015), Dielectric properties of Asteroid Vesta's surface as constrained by Dawn VIR observations, Icarus, 262, 93-101.

Pappalardo, R. T., et al. (2015), Science and reconnaissance from the Europa clipper mission concept: Exploring Europa's habitability, in 46th Lunar and Planetary Science Conference, vol. 1832, p. 2673, LPI Contribution, Woodlands, Tex.

Pätzold, M., et al. (2016), A homogeneous nucleus for 67P/Churyumov-Gerasimenko from its gravity field, Nature, 530, 63-65, doi:10.1038/nature16535.

Pettinelli, E., et al. (2015), Dielectric properties of Jovian satellite ice analogs for subsurface radar exploration: A review, Rev. Geophys., 53, 593-641, doi:10.1002/2014RG000463.

Pettinelli, E., et al. (2016), Dielectric characterization of ice/ $\mathrm{MgSO}_{4} \cdot 11 \mathrm{H}_{2} \mathrm{O}$ mixtures as Jovian icy moon crust analogues, Earth Planet. Sci. Lett., 439, 11-17.

Pilcher, C. B., S. T. Ridgeway, and T. B. McCord (1972), Galilean satellites: Identification of water frost, Science, 178, 1087-1089, doi:10.1126/science.178.4065.1087.

Poch, O., et al. (2016), Sublimation of water ice mixed with silicates and tholins: Evolution of surface texture and reflectance spectra, with implications for comets, Icarus, 267, 154-173.

Sabouroux, P., and D. Ba (2011), EPSIMU, a tool for dielectric properties measurement of porous media: Application in wet granular materials characterzation, Pr. Electromagn. Res., 29, 191-207.

Schloerb, F. P., et al. (2015), MIRO observations of subsurface temperatures of the nucleus of 67P/Churyumov-Gerasimenko, Astron. Astrophys., 583, A29.

Seidensticker, K. J., et al. (2007), Sesame - An experiment of the Rosetta Lander Philae: Objectives and general design, Space Sci. Rev., 128, $301-337$.

Seu, R., et al. (2004), SHARAD: The MRO 2005 shallow radar, Planet. Space Sci., 52, 157-166.

Sierks, H., et al. (2015), On the nucleus structure and activity of comet 67P/Churyumov-Gerasimenko, Science, 347, aaa1044, doi:10.1126/science.aaa 1044 .

Sihvola, A. H. (Ed.) (1999), Electromagnetic Mixing Formulas and Applications, vol. 47 of Electromagnetic Waves Serie, IEEE, London. Spohn, T., and G. Schubert (2003), Oceans in the icy Galilean satellites of Jupiter?, Icarus, 161, 456-467.

Stillman, D. E., R. E. Grimm, and S. F. Dec (2010), Low-frequency electrical properties of ice silicate mixtures, J. Phys. Chem. B, 114, 6065-6073. Ulaby, F. T., and D. G. Long (2014), Microwave Radar and Radiometric Remote Sensing, Univ. Michigan Press, Ann Arbor, Mich.

von Hippel, A., D. B. Knoll, and W. B. Westphal (1971), Transfers of protons through "Pure" ice lh single crystals. I. Polarization spectra of ice Ih, J. Chem. Phys., 54, 134.

Waite, A. H., and S. J. Schmidt (1962), Gross errors in height indication from pulsed radar altimeters operating over thick ice or snow, Proc. IRE, 50, 1515-1520.

Yoldi, Z., et al. (2015), VIS-NIR reflectance of water ice/regolith analogue mixtures and implications for the detectability of ice mixed within planetary regoliths, Geophys. Res. Lett., 42, 6205-6212, doi:10.1002/2015GL064780.

Zimmer, C., K. K. Khurana, and M. G. Kivelson (2000), Subsurface oceans on Europa and Callisto: Constraints from Galileo magnetometer observations, Icarus, 147, 329-347. 